\title{
WILEY-VCH
}

DOI: $10.1002 /(($ please add manuscript number $))$

Article type: Communication

\section{Solution-Grown Monocrystalline Hybrid Perovskite Films for Hole-Transporter Free Solar Cells}

Wei Peng, Lingfei Wang, Banavoth Murali, Kang-Ting Ho, Ashok Bera, Namchul Cho, ChenFang Kang, Victor M. Burlakov, Jun Pan, Lutfan Sinatra, Chun Ma, Wei Xu, Dong Shi, Erkki Alarousu, Alain Goriely, Jr-Hau He, Omar F. Mohammed, Tom Wu, and Osman M. Bakr*

W. Peng, Dr. L. Wang, Dr. B. Murali, Dr. N. Cho, Dr. J. Pan, L. Sinatra, Dr. D. Shi, Dr. E. Alarousu, Prof. O. F. Mohammed, Prof. O. M. Bakr

Division of Physical Sciences and Engineering, Solar and Photovoltaics Engineering Research Center (SPERC), King Abdullah University of Science and Technology (KAUST), Thuwal 23955-6900, Kingdom of Saudi Arabia

E-mail: osman.bakr@kaust.edu.sa

K.-T. Ho, Dr. C.-F. Kang, Prof. J.-H. He

Division of Computer, Electrical and Mathematical Sciences and Engineering (CEMSE),

King Abdullah University of Science \&Technology (KAUST), Thuwal 23955-6900,

Kingdom of Saudi Arabia

Dr. A. Bera, C. Ma, Prof. T. Wu

Materials Science and Engineering, Division of Physical Sciences and Engineering, King Abdullah University of Science and Technology (KAUST), Thuwal 23955-6900, Kingdom of Saudi Arabia

Dr. V. M. Burlakov, Prof. A. Goriely

Mathematical Institute, University of Oxford, Woodstock Road, Oxford OX2 6GG, U.K.

Dr. W. Xu

Imaging and Characterization Lab, King Abdullah University of Science and Technology

(KAUST), Thuwal 23955-6900, Kingdom of Saudi Arabia

Keywords: (hybrid perovskites, single crystals, hole-transporter free solar cells, $\mathrm{CH}_{3} \mathrm{NH}_{3} \mathrm{PbBr}_{3}$ )

Over the past few years, tremendous progress on perovskite solar cells (PSCs) has been achieved by improving the crystallinity of perovskite polycrystalline films. ${ }^{[1-5]}$ This promising trend may persist for some time, since polycrystalline films employed in the best PSCs are still orders of magnitude inferior to perovskite single crystals in terms of trap density, carrier mobility and carrier diffusion length. ${ }^{[6-10]}$ Furthermore, even if the optimization of processing conditions leads to considerably improved crystallinity, grain-scale inhomogeneity and 


\section{WILEY-VCH}

reliance on electron and hole transporting materials in state-of-the-art PSCs would result in several deficiencies in terms of stability, cost and manufacturability. ${ }^{[1-13]}$ Perovskite monocrystalline (i.e. single crystal) films, which are free of grain boundaries, can achieve the upper bounds of lifetimes and diffusion lengths for all carriers homogeneously across the perovskite layer, ${ }^{[11]}$ and thus enable the engineering of PSCs with a single junction to efficiently separate and collect photocarriers. ${ }^{[14]}$ A simpler device architecture based on a monocrystalline film may provide a potential solution for overcoming the challenges in the development of PSCs. Unfortunately, single crystal perovskites are mostly grown in bulk and have yet to be realized in the form of films, ${ }^{[6-8]}$ which is an essential prerequisite for practical device applications. The hybrid nature of organometal halide perovskites, i.e. intercalation of volatile and vulnerable organic components with a brittle inorganic framework, makes their monocrystalline films difficult to be achieved by deposition techniques that are wellestablished for inorganic compounds (e.g., pulsed laser deposition and sputtering) and organic materials (e.g., vapor growth) ${ }^{[15-17]}$

We were thus motivated to grow hybrid perovskite monocrystalline films on substrates by exploiting the perovskite's propensity to crystallize in solution. However, simple solutionbased techniques that crystallize perovskites from saturated precursor solutions produce only free-standing bulk crystals with small aspect ratios. ${ }^{[6-8]}$ Such geometries are unsuitable for optoelectronic devices from perspectives of both dimensions and device fabrications, especially since the deposition conditions of transparent conducting oxides, which are integral components for most optoelectronic devices, generally preclude their direct deposition on perovskite crystals. For example, $\mathrm{CH}_{3} \mathrm{NH}_{3} \mathrm{PbBr}_{3} \quad\left(\mathrm{MAPbBr}_{3}\right)$ crystals grown from supersaturated solutions, such as by slow diffusion of antisolvent vapor into the perovskite solution, were several millimeters in cubic shape and resisted growing on substrates (Figure S1, Supporting Information). Likely, the disinclination to heterogeneously nucleate on 


\section{WILEY-VCH}

substrate surfaces resulted from the higher nucleation energy barrier of smooth surfaces. ${ }^{[18]}$ Therefore, we designed a new growth method based on a cavitation-triggered asymmetrical crystallization (CTAC) strategy, which would promote heterogeneous nucleation by providing enough energy to overcome the nucleation barrier. Briefly, we introduced a very short ultrasonic pulse $(\sim 1 \mathrm{~s})$ to the solution when it reached a low supersaturation level with antisolvent vapor diffusion (See Experimental Session). Promisingly, $\mathrm{MAPbBr}_{3}$ monocrystalline films grew on the surface of various substrates within several hours of the ultrasonic pulse (Figure S1, Supporting Information). As shown in the photographic image (Figure 1a), as well as the cross-sectional (Figure 1b) and top-view (Figure S2) scanning electron microscopy (SEM) images, these semitransparent films are homogeneous and free of grain boundaries, with thicknesses varying from one up to several tens of micrometers, and lateral dimensions ranging from hundreds of microns to three millimeters. We should note here that growing $\mathrm{MAPbI}_{3}$ monocrystalline films is more challenging due to the large difference in the solubility of $\mathrm{MAI}$ and $\mathrm{PbI}_{2}$ and possibly the intrinsic anisotropic growth of tetragonal crystals, which may naturally not prefer the growth direction along the substrates.

Since the first report on the application of ultrasound in crystallization in $1927,{ }^{[19]}$ the influence of ultrasound on nucleation has been extensively studied. ${ }^{[20]}$ Ultrasound was found to promote nucleation under a low supersaturation level without the aid of seed crystals. The role of ultrasound in the nucleation process was assumed to work through a cavitation process, where successive cycles of compression and rarefaction sound waves create and collapse cavities, repeatedly. The release of transient ultrahigh energy that typically accompanies the cavitation process is expected to induce nucleation by overcoming the nucleation barrier through rapid local cooling rates, increasing local pressure and accumulating energy to overcome the nucleation barrier. ${ }^{[21]}$ The collapse of cavities is asymmetric near a solid surface and generates high-speed jets of fluid towards the surface, known as micro-jetting. ${ }^{[22]}$ This 


\section{WILEY-VCH}

process has been utilized for film thinning and reducing particle sizes as a result of the induced strong shearing force,${ }^{[23]}$ which may also contribute to the asymmetric crystal growth we observed here. Figure 1c-f illustrates the entire CTAC process. However, the situation is dramatically different when the ultrasonic pulse is introduced at high supersaturation levels, where cavitation triggers excessive nucleation events and therefore the growth of a plethora of tiny crystals (Figure S1, Supporting Information).

Due to the physical nature of cavitation, the growth of monocrystalline films by CTAC is largely independent of substrate properties. Hence, we were able to grow monocrystalline perovskite films on various substrates such as silicon wafers, indium tin oxide (ITO)-coated glass, fluorine-doped tin oxide (FTO)-coated glass and sputter-coated metal on silicon (Figure S1, Supporting Information). The cross-sectional SEM (Figure 1b) confirms a good mechanical contact at the crystal/substrate interface by showing no gaps existing at the interface, which is further proved to be a good electrical contact by the electrical characterizations shown below. We also noticed the dependence of monocrystalline film thicknesses on precursor concentrations (see Figure S3, Supporting Information). These findings underscore the value of our solution-based CTAC method for enabling perovskite monocrystalline film-based optoelectronic devices, despite the coarse control over the film thickness. But we believe that the control over film thickness can be further refined by precisely regulating the rate and time of crystal nucleation and growth, the moment to introduce sonication, post-sonication growth time and antisolvent engineering,.

High-resolution X-ray diffraction (HRXRD) was used to assess the phase purity and single crystal nature of the films. The $\omega-2 \theta$ diffraction pattern of powder from crushed as-grown crystals (Figure 2a) confirmed the exclusive presence of the cubic $\mathrm{MAPbBr}_{3}$ phase. The $\omega-2 \theta$ scan of the film (Figure $2 \mathrm{~b}$ ) shows only $\{001\}$ diffractions, and the $\varphi$-scan of (110)-diffraction $\left(2 \theta=21.42^{\circ}, \psi=45^{\circ}\right)$ shows a four-fold symmetry (Figure $2 \mathrm{c}$ ), clearly confirming that the 


\section{WILEY-VCH}

film is a (001)-oriented, twin-free single crystal with cubic symmetry. We then checked the rocking curve of the (002) diffraction (Figure 2d), and measured a full width at half maximum (FWHM) of $0.040^{\circ}$. As a qualitative indication of crystallinity, this value is comparable to those of high-quality epitaxial perovskite oxide films. ${ }^{[16,24]}$ Notably, it is also smaller than that of bulk $\mathrm{MAPbBr}_{3}$ perovskite crystals $\left(\mathrm{FWHM}=0.044^{\circ}\right.$, Figure $\left.2 \mathrm{~d}\right)$, which suggests that the cavitation process, though as an external disturbance, does not deteriorate crystallinity. The high quality of the monocrystalline films was further corroborated by the space-charge limited current measurement, showing a low trap density of $\sim 10^{11} \mathrm{~cm}^{-3}$ (Figure S4, Supporting Information).

Monocrystalline films grown by the CTAC method had thicknesses within the range of carrier diffusion lengths that have been previously measured for bulk $\mathrm{MAPbBr}_{3}$ single crystals. ${ }^{[7]}$ It is thus reasonable to expect that efficient collection of photocarriers could be achieved in photovoltaic devices comprising these films. Therefore, we fabricated solar cells by simply evaporating gold electrodes onto the surface of monocrystalline films grown on ITO-coated glass (see Methods). The device architecture is illustrated in the inset of Figure $\mathbf{3 d}$ and photos of real devices are shown in Figure S6, Supporting Information. For photovoltaic characterizations, a bias was applied through the Au electrode and the ITO was placed under illumination (air mass 1.5 (AM 1.5) and $100 \mathrm{~mW} \mathrm{~cm}^{-2}$ power). By photoelectron spectroscopy in air (PESA), we determined the work function of the monocrystalline films to be $\sim 5.63 \mathrm{eV}$ (see Figure S7, Supporting Information). This finding suggests the presence of a large Schottky barrier at the ITO/perovskite interface, considering a work function of $\sim 4.6 \mathrm{eV}$ for ITO. In contrast, the contact of perovskite/Au is expected to be Ohmic due to the large work function of $\mathrm{Au}(5.1-5.4 \mathrm{eV})$, which is also consistent with the linear character of the $I-V$ curves of a lateral Au/perovskite/Au structure (Figure S4b, Supporting Information). The band alignment is thus as presented in Figure 3a. The direction of the Schottky diode is 


\section{WILEY-VCH}

confirmed by the apparent current rectification of the dark current density-voltage $(J-V)$ curve (Figure S8, Supporting Information). These findings suggest the $p$-type nature of the $\mathrm{MAPbBr}_{3}$ monocrystalline films we grow.

Figure 3c shows the illuminated $J-V$ curves of devices with $\mathrm{MAPbBr}_{3}$ monocrystalline films of various thicknesses. Detailed photovoltaic parameters of these cells can be found in Table 1. Histograms of photovoltaic parameters for 12 devices based on different $4-\mu \mathrm{m}$ thick films are shown in Figure S9, Supporting Information. The statistical data of power conversion efficiencies (PCEs) shows a very small deviation $(0.2 \%)$ with an average of $5.00 \%$. The best cell shows a fill factor (FF) of 0.58 , an open-circuit voltage $\left(V_{\mathrm{OC}}\right)$ of $\sim 1.24 \mathrm{~V}$ and a remarkable short-circuit current $\left(J_{S C}\right)$ of $\sim 7.42 \mathrm{~mA} \mathrm{~cm}^{-2}$, yielding a PCE of $5.37 \%$. The large $J_{\mathrm{SC}}$ value agrees well with a high and wide plateau of the device's external quantum efficiency (EQE) spectrum, as shown in Figure 3e, from which we calculated an integrated current density of $7.15 \mathrm{~mA} \mathrm{~cm} \mathrm{~cm}^{-2}$. The corresponding internal quantum efficiency (IQE), calculated by dividing the EQE by the transmittance of ITO-coated glass (Figure S10, Supporting Information), shows an onset at $\sim 550 \mathrm{~nm}$ with a plateau higher than $90 \%$ over a wide spectral range and a peak value of $\sim 97 \%$ at $507 \mathrm{~nm}$ (Figure 3e). Moreover, we calculated the integrated current's theoretical upper limit to be $7.64 \mathrm{~mA} \mathrm{~cm}{ }^{-2}$, by assuming a $100 \%$ IQE and that the only loss of photons is through absorption and reflection by ITO glass substrates (Figure S10, Supporting Information). The small difference between the experimental value and the theoretical upper limit confirms the high quantum efficiency of our devices. In addition, the $J_{\mathrm{SC}}$ value is comparable with those of the best polycrystalline tribromide PSCs reported in the literature. ${ }^{[25]}$

To understand the origin of the near-unity IQE achieved in our simple ITO/MAPbBr $3 / \mathrm{Au}$ structure, we sought to clarify the working principles of the cells. The capacitance-voltage $(C$ $V)$ measurement was carried out to examine the built-in potential existing in the device. ${ }^{[26]} \mathrm{A}$ 


\section{WILEY-VCH}

built-in potential of $\sim 1.2 \mathrm{~V}$ was extracted from the Mott-Schottky plot of the $C-V$ measurement (Figure S11, Supporting Information), which is in good agreement with the measured $V_{\mathrm{OC}}$. We further calculated a self-dopant concentration of $\sim 10^{12} \mathrm{~cm}^{-3}$ from the slope of the linear region of the plot. Accordingly, the depletion width was calculated to be $\sim 7 \mu \mathrm{m}$ (See Supporting Information). This large depletion width, mainly a consequence of the low self-dopant concentration, would ensure the full depletion of electrons within the $4 \mu \mathrm{m}$-thick crystal. Under such a built-in field that spans across the perovskite film, photo-excited electrons could easily drift to the ITO electrode and be collected due to the small absorption depth. Complementary to the extraction of electrons, photo-excited holes are easily collected at the $\mathrm{Au}$ electrode because of the Ohmic contact of $\mathrm{MAPbBr}_{3} / \mathrm{Au}$. Note that such working principles are inferred based on the fact that excitons dissociate immediately after generation. $^{[14]}$

Considering the discussion above, a major part of the perovskite crystal that is located more than an absorption depth away from the ITO acts as a carrier transporter rather than as a light absorber (Figure 3a). Therefore, if this part of the perovskite film is too thick, it would considerably increase the series resistance and decrease the FF, which is evident in the $J-V$ curves measured from devices with crystal thicknesses increasing from 4 to $12 \mu \mathrm{m}$ (Figure $3 \mathrm{c}$ ). However, by further reducing the thickness to $1 \mu \mathrm{m}$, no significant improvement was observed in the FF, as well as $V_{\mathrm{OC}}, J_{\mathrm{SC}}$ and PCE (see Figure $3 \mathrm{c}$ and Table 1). This trend is reasonable since the resistance is not substantially reduced with the reduction of the crystal thickness from $4 \mu \mathrm{m}$ to $1 \mu \mathrm{m}$. Furthermore, it is worth pointing out that there is no apparent loss of $J_{\mathrm{SC}}$ with increasing crystal thickness up to $12 \mu \mathrm{m}$, due to the long diffusion length of $\sim 10 \mu \mathrm{m}$ and a large depletion width of $\sim 7 \mu \mathrm{m}$ under short-circuit condition. On the other hand, $V_{\mathrm{OC}}$ significantly decreased with increasing the crystal thickness because of the increased carrier recombination probability under working conditions near $V_{\mathrm{OC}}$. When the 


\section{WILEY-VCH}

monocrystalline film thickness was increased to $60 \mu \mathrm{m}$, which is far beyond the depletion width and the charge-carrier diffusion length, the current was severely reduced $\left(J_{\mathrm{SC}}=2.2 \mathrm{~mA}\right.$ $\mathrm{cm}^{-2}$ ) as a result of the substantially amplified recombination rate.

To determine whether the Schottky-junction based cell structure could be applied to polycrystalline films, a sequential evaporation method was used to deposit pinhole-free $\mathrm{MAPbBr}_{3}$ polycrystalline films (see Experimental Session). Although SEM images (Figure S12a and b, Supporting Information) confirm the uniform morphology and complete coverage of as-grown films, we could only avoid device shorting and obtain rectified currents when the thickness of the polycrystalline perovskite layer was larger than $\sim 1 \mu \mathrm{m}$ (Figure S12c, Supporting Information) - likely due to defects or grain boundary-driven leakage paths forming in the thin polycrystalline perovskite layer. As shown in Figure S12d, Supporting Information, the solar cell based on a $1 \mu \mathrm{m}$-thick polycrystalline film shows a $J_{\mathrm{SC}}$ of $0.59 \mathrm{~mA}$ $\mathrm{cm}^{-2}$, a $V_{\mathrm{OC}}$ of $0.29 \mathrm{~V}$, and an FF of 0.33 , yielding a PCE of $0.056 \%$. Although the polycrystalline film was comprised of nearly a monolayer of grains, the poor performance indicates that polycrystalline films may not be suitable for such a simple Schottky-junction based cell structure, possibly due to the existence of grain boundaries. ${ }^{[11]}$

The promising efficiencies we have achieved in the Schottky-junction based device structure were obtained primarily by optimizing the thickness of the perovskite layer. The performance of solar cells, however, is well known to be sensitive to the interface properties. ${ }^{[27,28]} \mathrm{We}$ were thus driven to further investigate the potential of enhancing the performance of monocrystalline film-based devices by modifying those properties. We therefore examined a single $p$ - $n$ junction based cell structure, i.e. $\mathrm{FTO} / \mathrm{TiO}_{2} / \mathrm{MAPbBr}_{3} / \mathrm{Au}$, where a compact $\mathrm{TiO}_{2}$ film acts as an electron transporting layer (ETL), as displayed in Figure 3b. The existence of a $\mathrm{p}-\mathrm{n}$ junction at the $\mathrm{TiO}_{2} / \mathrm{MAPbBr}_{3}$ interface is corroborated by the clear current rectification in the $J-V$ curve of the cell (Figure S8, Supporting Information). 


\section{WILEY-VCH}

With such a structure, we managed to enhance the PCE of our monocrystalline devices to $\sim 6.53 \%$, with a $V_{\mathrm{OC}}$ of $1.36 \mathrm{~V}$, a $J_{\mathrm{SC}}$ of $6.96 \mathrm{~mA} \mathrm{~cm} \mathrm{~cm}^{-2}$ and an $\mathrm{FF}$ of 0.69 , for a champion device made of a $1 \mu \mathrm{m}$-thick perovskite crystal. This device performance surpasses, by over $17 \%$, other hole-transporting layer (HTL)-free polycrystalline $\mathrm{MAPbBr}_{3}$ solar cells reported to date, ${ }^{[29,30]}$ and is even comparable to those of devices with a HTL. ${ }^{[25,31]}$ The average efficiency of 12 monocrystalline devices using this structure with 1 to $4 \mu \mathrm{m}$ thick crystals is $5.67 \pm 0.44 \%$ (Figure S9, Supporting Information). The corresponding EQE and IQE curves are presented in Figure 3e, showing a similar plateau as those of Schottky junction-based devices, with exception of being inferior at wavelengths below $350 \mathrm{~nm}$ due to stronger UV absorption of $\mathrm{FTO} / \mathrm{TiO}_{2}$ substrates, resulting in a decreased $J_{\mathrm{SC}}$. The current density integrated from the EQE curve is $6.7 \mathrm{~mA} \mathrm{~cm}^{-2}$, in good agreement with the value extracted from the $J-V$ curve, which is also approaching the theoretical upper limit of $7.24 \mathrm{~mA} \mathrm{~cm}{ }^{-2}$ (Figure S10, Supporting Information). A notable feature of this single p-n junction-based monocrystalline device is the greatly improved $V_{\mathrm{OC}}$ (the highest reached so far was $1.41 \mathrm{~V}$, see Figure S9, Supporting Information). This is in agreement with the energy difference between the Fermi levels of $\mathrm{TiO}_{2}$ and $\mathrm{MAPbBr}_{3}(\Delta E \sim 1.4 \mathrm{eV})$, which is larger than that between ITO and $\mathrm{MAPbBr}_{3}$. The $V_{\mathrm{OC}}$ is also the highest among reported $\mathrm{MAPbBr}_{3}$ solar cells without a HTL, ${ }^{[29,30]}$ and is on par with the best performing cells with a custom-designed HTL. ${ }^{[25]}$ Such improvements clearly imply that the $\mathrm{TiO}_{2} /$ perovskite-based devices extract electrons more efficiently than ITO/perovskite based devices. With such demonstrably high $V_{\mathrm{OC}}$ and $J_{\mathrm{SC}}$, we believe there is tremendous potential using perovskite monocrystalline films in the HTL-free device structures, in which there is large for further improvements through interface engineering approaches such as chemical passivation. ${ }^{[1]}$

Note that we found considerable hysteresis in the $J-V$ curves of $\mathrm{TiO}_{2}$-free devices. (Figure 4a). In contrast, the hysteresis was substantially reduced in the devices with a $\mathrm{TiO}_{2}$ layer 


\section{WILEY-VCH}

(Figure 4b). We further confirmed the PCE of our devices by measuring the steady-state maximum power output, which was determined through measuring the transient current output at the bias of the device's maximum power point $\left(V_{\max }\right) \cdot{ }^{[32,33]}$ As displayed in Figure 4c, the ITO device made with a $4 \mu \mathrm{m}$-thick crystal and $\mathrm{FTO} / \mathrm{TiO}_{2}$ device made with a $1 \mu \mathrm{m}$ thick crystal show stable PCEs of $5.11 \%$ and $6.33 \%$, respectively, at their corresponding $V_{\max }$, which were determined from their reversely scanned $J-V$ curves. The steady-state photocurrents of the ITO device shown in Figure S13 (Supporting Information) at various biases near $V_{\max }$ further verify that reversely scanned $J-V$ curves provide a reliable estimation of the true device performance.

Several theories have been proposed to explain the origin of hysteresis observed in PSCs. ${ }^{[32,34-36]}$ Here, the PCE obtained from the reversely scanned $J-V$ curve is close to that extracted from the transient current measurement, which agrees well with the trapping-detrapping model. ${ }^{[32]}$ Although $\mathrm{MAPbBr}_{3}$ crystals have an ultra-low bulk trap density, ${ }^{[7]}$ defects and trap states could accumulate at the crystal surface, which is also supported by the PL study (Figure S5, Supporting Information). In addition, labile ions may accumulate in the disordered perovskite lattice in the vicinity of contacts and can drift under operational conditions, resulting in a slow process of electrode polarization ${ }^{[35]}$ or screening of space charges ${ }^{[32]}$ for efficient photocarrier collection. Based on the discussion above, the $\mathrm{TiO}_{2}$ interlayer could have minimized the traps of the perovskite bottom surface, resulting in less hysteresis, which is further corroborated by a faster stabilization of transient photocurrent, as shown in Figure $4 \mathrm{c}$.

Device stability, a present-day bottle-neck in the commercialization of PSCs, was expected to improve in our simple-structured solar cells through removing components that may cause instability issues. $^{[13,37,38]}$ Hence, we tested the stability of these cells by monitoring the photocurrent of the monocrystalline cell while continuously exposed to simulated AM1.5 


\section{WILEY-VCH}

sunlight $\left(100 \mathrm{~mW} \mathrm{~cm}^{-2}\right)$ in an ambient atmosphere (55\% relative humidity, room temperature), without encapsulation. Strikingly, the photocurrent of the Schottky-junction based device shows no degradation under $V_{\max }$ in our test of over 48 hours, while the current of the monocrystalline device using $\mathrm{TiO}_{2}$ shows slight degradation and drops to $\sim 90 \%$ of its initial level (Figure 4d). For comparison, we fabricated two polycrystalline cells: one with the same structure as the Schottky-junction based monocrystalline cell (Poly-1); and the other with both a HTL and a ETL in a mesostructure of $\mathrm{FTO} / \mathrm{TiO}_{2} / \mathrm{MAPbBr}_{3} / 2,2^{\prime}, 7,7^{\prime}$-tetrakis[N,N-di(4methoxyphenyl)amino]-9,9'-spirobifluorene (Spiro-OMeTAD)/Au (Poly-2). The normalized current of both cells shows distinct degradation at different rates. $J-V$ curves of the four cells before and after illumination are provided in Figure S12, S14 and S15, Supporting Information. The superior stability of monocrystalline devices, especially the one based on Schottky junction, over polycrystalline film PSCs may have several origins. First, hybrid perovskite polycrystalline films have been reported to degrade fast in the presence of moisture, while perovskite single crystals have been found to be stable for over one month in ambient conditions. ${ }^{[6]}$ Compared with polycrystalline films, monocrystalline films are free of grain boundaries, which are supposed to be the infiltration and corrosion sites for moistures. Second, the absence of a $\mathrm{TiO}_{2}$ layer may eliminate potential instability factors such as light-induced desorption of surface-adsorbed oxygen, ${ }^{[13]}$ which can also account for the stability difference between the two monocrystalline device architectures. And finally, the organic HTL, which is absent in monocrystalline cells, has been known to cause instability issues for PSCs under exposure to moisture and oxygen. ${ }^{[37,38]}$ The two latter factors may explain the difference in current degradation rates between the two polycrystalline solar cells.

In summary, we reported the first successful growth and characterization of hybrid perovskite monocrystalline films on substrates, achieved only by using a method that we term CTAC, which overcomes the shortcomings of traditional single crystal growth methods in 


\section{WILEY-VCH}

their tendency to produce merely free-standing perovskite single crystals. Therefore, we were able to carry out the first-reported explorative study on perovskite monocrystalline solar cells using two different device structures. Without any ETLs and HTLs, a simple prototype cell of ITO/MAPbBr $3(4 \mu \mathrm{m}) / \mathrm{Au}$ offers near-unity IQE, and consequently an ultra-stable PCE of $>$ 5\%. Furthermore, by employing a single p-n junction based architecture instead, we enhanced the efficiency to $6.5 \%$, which is a $>17 \%$ improvement over the best HTL-free $\mathrm{MAPbBr}_{3}$ solar cells. Remarkably, this simple device structure offers a high $V_{\mathrm{OC}} \sim 1.4 \mathrm{~V}$, which makes it particularly attractive for applications in photoelectrocatalytic water-splitting systems for solar fuels ${ }^{[39]}$ and also as top cells in tandem solar cells with Si and CIGS. We elucidated the roles of the monocrystalline film thickness and interface engineering, and their crucial effects on photocarrier collection in single crystal solar cells. Our work provides direct proof for the superior optoelectronic properties of perovskite monocrystalline films in devices over their polycrystalline counterparts. Moreover, the demonstration of extraordinary stability of Schottky-junction based monocrystalline devices, along with its fabrication simplicity, opens a new avenue for the development of perovskite solar cells, akin to the evolution of metalinsulator-semiconductor inversion-layer silicon solar cells. ${ }^{[40]}$

\section{Experimental Section}

Materials: MABr powder and $\mathrm{TiO}_{2}$ paste were purchased from Dyesol. Spiro-OMeTAD was purchased from Borun New Material Technology. All the other chemicals and solvents were purchased from Sigma Aldrich and are ACS reagent grade. ITO-coated glass substrates (8-12 $\left.\Omega \mathrm{sq}^{-1}\right)$ were purchased from Sigma Aldrich. FTO-coated glass substrates $\left(15 \Omega \mathrm{sq}^{-1}\right)$ were purchased from Pilkington.

Monocrystalline film growth: The experimental set-up for the crystal growth was previously reported. Briefly, perovskite precursor solution of equimolar $\mathrm{MABr}$ and $\mathrm{PbBr}_{2}$ in $\mathrm{N}, \mathrm{N}-$ dimethylformamide (DMF, typically $10 \mathrm{~mL}$ ), was loaded in a Pyrex crystallizing dish $(80 \mathrm{~mm}$ 


\section{WILEY-VCH}

$\times 40 \mathrm{~mm}$ ) with substrates placed in the bottom, which was then placed in a larger crystallizing dish $(125 \mathrm{~mm} \times 65 \mathrm{~mm})$ with dichloromethane loaded as antisolvent. Both crystallizing dishes were sealed with aluminum foils while the cover of the inner dish was left with a $0.5 \mathrm{~mm}$ diameter hole to let the antisolvent vapor slowly diffuse into the perovskite solution. To grow monocrystalline films, the whole set-up was transferred to an ultrasonic bath (Branson 5510) and a short ultrasonic pulse $(<1 \mathrm{~s})$ was triggered when the perovskite solution reached a low supersaturation level, which was roughly estimated to be 3-6 hours in advance of the moment that crystals came out of the solution in the absence of ultrasound.

Monocrystalline film characterization: SEM images were taken with a Quanta 600 FEG (FEI Co.). XRD measurements were carried out by Panalytical X'pert Pro equipped with a $\mathrm{Cu} \mathrm{K}_{\alpha}$ radiation source $(\lambda=1.5406 \AA)$. The absorption spectra were captured with a Varian Cary 6000i spectrometer in a transmittance mode. Aramis Raman Spectroscopy (Horiba Scientific, Japan) was used to record the photoluminescence spectra excited by a $473 \mathrm{~nm}$ laser in a $180^{\circ}$ reflection setup. The thickness of the crystal was measured by a KLA Tencor Stylus Profilometer. PESA measurement was carried out on $\mathrm{MAPbBr}_{3}$ monocrystalline films grown on glass using Riken Photoelectron Spectrometer (Model AC-2). The UV lamp intensity was fixed at $50 \mathrm{nW}$, which was pre-calibrated for the light correction.

Device fabrication: We fabricated four types of solar cells with three different configurations in this work. For the monocrystalline device, $100 \mathrm{~nm}$ Au electrodes of a circular shape (390 $\mu \mathrm{m}$ in diameter) or full coverage of $\mathrm{Au}$ on a whole monocrystalline film were deposited through metal shadow mask onto $\mathrm{MAPbBr}_{3}$ monocrystalline films that were grown on ITOcoated glass or $\mathrm{TiO}_{2}(\sim 60 \mathrm{~nm}) /$ FTO glass by thermal evaporation. The efficiencies for different Au patterns were similar and a high efficiency was achieved on a $\sim 2 \times 2 \mathrm{~mm}^{2}$ monocrystalline film (see Figure S6, Supporting Information). The deposition of compact $\mathrm{TiO}_{2}$ layers was as reported elsewhere. ${ }^{[41]}$ For the third type of PSCs, by using the same configuration of ITO based monocrystalline device, a polycrystalline $\mathrm{MAPbBr}_{3}$ film was 


\section{WILEY-VCH}

instead deposited on ITO-coated glass by a two-step vapor deposition method. $\mathrm{A} \mathrm{PbBr}_{2}$ film was thermally evaporated onto ITO-coated glass and annealing in MABr vapor at $130{ }^{\circ} \mathrm{C}$ under vacuum for a certain time in a vacuum desiccator followed to convert $\mathrm{PbBr}_{2}$ into perovskite. The thickness of the final perovskite film was controlled through the initial $\mathrm{PbBr}_{2}$ film thickness. Finally, Au electrodes of the same geometry as monocrystalline devices were evaporated on top. The mesostructured device was fabricated by sequential deposition of a compact $\mathrm{TiO}_{2}$ layer, a $200 \mathrm{~nm}$ mesoporous $\mathrm{TiO}_{2}$ layer, $\mathrm{MAPbBr}$, Spiro-OMeTAD and 100 $\mathrm{nm}$ Au electrodes on patterned FTO-coated glass. Specifically for the deposition of $\mathrm{MAPbBr}_{3}$ film, a solution of $40 \mathrm{wt} \%$ perovskite precursor was prepared with equimolar $\mathrm{MABr}$ and $\mathrm{PbBr}_{2}$ in DMF at $60{ }^{\circ} \mathrm{C}$ stirring overnight and then adding $\mathrm{HBr}(20 \mu \mathrm{L}, 48 \mathrm{wt} \%$ in water $)$. $\mathrm{MAPbBr}_{3}$ film was deposited by spin-coating the as-prepared solution at $3000 \mathrm{rpm}$ for $3 \mathrm{~min}$ and annealed at $100{ }^{\circ} \mathrm{C}$ for $10 \mathrm{~min}$. The detailed procedures for depositing the compact/mesoporous $\mathrm{TiO}_{2}$ layer and Spiro-OMeTAD layer were published elsewhere. ${ }^{[41]}$ The active device area is $0.2 \mathrm{~cm}^{2}$.

Device characterization: To characterize cell performance, simulated air-mass 1.5 (AM1.5) sunlight with a power intensity of $100 \mathrm{~mW} \mathrm{~cm}^{-2}$ (Newport Oriel Sol3A) was used as the light source. $J-V$ curves were recorded with a Keithley 4200 sourcemeter with a scan rate of $0.2 \mathrm{~V}$ $\mathrm{s}^{-1}$ with the same mask for thermal evaporation used as aperture mask for small devices. The EQE and IQE curves were measured by a Newport Oriel IQE-200 system equipped with a $300 \mathrm{~W}$ xenon light source, a monochromator and a Keithley 2400 sourcemeter. The capacitance-voltage ( $C$ - $V$ ) curve was measured by Agilent E4980A precision LCR meter. 


\section{WILEY-VCH}

\section{Supporting Information}

Supporting Information is available from the Wiley Online Library or from the author.

\section{Acknowledgements}

This research is supported by King Abdullah University of Science and Technology (KAUST).

W. Peng and L. Wang contributed equally to this work.

Received: ((will be filled in by the editorial staff))

Revised: ((will be filled in by the editorial staff)) Published online: ((will be filled in by the editorial staff))

[1] M. M. Lee, J. Teuscher, T. Miyasaka, T. N. Murakami, H. J. Snaith, Science 2012, 338, 643.

[2] J. Burschka, N. Pellet, S. J. Moon, R. Humphry-Baker, P. Gao, M. K. Nazeeruddin, M. Gratzel, Nature 2013, 499, 316.

[3] M. Liu, M. B. Johnston, H. J. Snaith, Nature 2013, 501, 395.

[4] N. J. Jeon, J. H. Noh, Y. C. Kim, W. S. Yang, S. Ryu, S. I. Seok, Nat. Mater. 2014, 13, 897.

[5] W. Nie, H. Tsai, R. Asadpour, J.-C. Blancon, A. J. Neukirch, G. Gupta, J. J. Crochet, M. Chhowalla, S. Tretiak, M. A. Alam, H.-L. Wang, A. D. Mohite, Science 2015, 347, 522.

[6] Q. Dong, Y. Fang, Y. Shao, P. Mulligan, J. Qiu, L. Cao, J. Huang, Science 2015, 347, 967.

[7] D. Shi, V. Adinolfi, R. Comin, M. Yuan, E. Alarousu, A. Buin, Y. Chen, S. Hoogland, A. Rothenberger, K. Katsiev, Y. Losovyj, X. Zhang, P. A. Dowben, O. F. Mohammed, E. H. Sargent, O. M. Bakr, Science 2015, 347, 519.

[8] M. I. Saidaminov, A. L. Abdelhady, B. Murali, E. Alarousu, V. M. Burlakov, W. Peng, I. Dursun, L. Wang, Y. He, G. Maculan, A. Goriely, T. Wu, O. F. Mohammed, O. M. Bakr, Nat. Commun. 2015, 6, 7586.

[9] S. D. Stranks, G. E. Eperon, G. Grancini, C. Menelaou, M. J. Alcocer, T. Leijtens, L. M. Herz, A. Petrozza, H. J. Snaith, Science 2013, 342, 341.

[10] G. Xing, N. Mathews, S. Sun, S. S. Lim, Y. M. Lam, M. Gratzel, S. Mhaisalkar, T. C. Sum, Science 2013, 342, 344. 


\section{WILEY-VCH}

[11] D. W. deQuilettes, S. M. Vorpahl, S. D. Stranks, H. Nagaoka, G. E. Eperon, M. E. Ziffer, H. J. Snaith, D. S. Ginger, Science 2015, 348, 683.

[12] A. Mei, X. Li, L. Liu, Z. Ku, T. Liu, Y. Rong, M. Xu, M. Hu, J. Chen, Y. Yang, M. Gratzel, H. Han, Science 2014, 345, 295.

[13] T. Leijtens, G. E. Eperon, S. Pathak, A. Abate, M. M. Lee, H. J. Snaith, Nat. Commun. 2013, 4, 2885.

[14] S. D. Stranks, H. J. Snaith, Nat. Nanotechnol. 2015, 10, 391.

[15] J. Wang, J. B. Neaton, H. Zheng, V. Nagarajan, S. B. Ogale, B. Liu, D. Viehland, V. Vaithyanathan, D. G. Schlom, U. V. Waghmare, N. A. Spaldin, K. M. Rabe, M. Wuttig, R. Ramesh, Science 2003, 299, 1719.

[16] C. B. Eom, R. J. Cava, R. M. Fleming, J. M. Phillips, R. B. vanDover, J. H. Marshall, J. W. P. Hsu, J. J. Krajewski, W. F. Peck, Science 1992, 258, 1766.

[17] C. Reese, Z. N. Bao, Mater. Today 2007, 10, 20.

[18] J. J. De Yoreo, P. G. Vekilov, Rev. Mineral. Geochem. 2003, 54, 57.

[19] A. P. Kapustin, The effects of ultrasound on the kinetics of crystallization, Springer Science \& Business Media, 2012.

[20] L. H. Thompson, L. K. Doraiswamy, Ind. Eng. Chem. Res. 1999, 38, 1215.

[21] G. Ruecroft, D. Hipkiss, T. Ly, N. Maxted, P. W. Cains, Org. Process Res. Dev. 2005, 9, 923.

[22] M. S. Plesset, R. B. Chapman, J. Fluid Mech. 1971, 47, 283.

[23] S. A. Elder, J. Acoust. Soc. Am. 1959, 31, 54.

[24] L. F. Wang, X. L. Tan, P. F. Chen, B. W. Zhi, B. B. Chen, Z. Huang, G. Y. Gao, W. B. Wu, AIP Adv. 2013, 3, 052106.

[25] J. H. Heo, D. H. Song, S. H. Im, Adv. Mater. 2014, 26, 8179.

[26] A. Guerrero, E. J. Juarez-Perez, J. Bisquert, I. Mora-Sero, G. Garcia-Belmonte, Appl. Phys. Lett. 2014, 105, 133902.

[27] Zhou, H.; Chen, Q.; Li, G.; Luo, S.; Song, T. B.; Duan, H. S.; Hong, Z.; You, J.; Liu, Y.; Yang, Y. Science 2014, 345, 542. 


\section{WILEY-VCH}

[28] Chen, Q.; Zhou, H.; Song, T.-B.; Luo, S.; Hong, Z.; Duan, H.-S.; Dou, L.; Liu, Y.; Yang, Y. Nano Letters 2014.

[29] A. Dymshits, A. Rotem, L. Etgar, J. Mater. Chem. A 2014, 2, 20776.

[30] H. B. Kim, I. Im, Y. Yoon, S. D. Sung, E. Kim, J. Kim, W. I. Lee, J. Mater. Chem. A 2015, 3, 9264.

[31] S. Ryu, J. H. Noh, N. J. Jeon, Y. Chan Kim, W. S. Yang, J. Seo, S. I. Seok, Energy Environ. Sci. 2014, 7, 2614.

[32] H. J. Snaith, A. Abate, J. M. Ball, G. E. Eperon, T. Leijtens, N. K. Noel, S. D. Stranks, J. T. W. Wang, K. Wojciechowski, W. Zhang, J. Phys. Chem. Lett. 2014, 5, 1511.

[33] J. A. Christians, J. S. Manser, P. V. Kamat, J. Phys. Chem. Lett. 2015, 6, 852.

[34] R. S. Sanchez, V. Gonzalez-Pedro, J. -W. Lee, N. -G. Park, Y. S. Kang, I. Mora-Sero, J. Bisquert, J. Phys. Chem. Lett. 2014, 5, 2357.

[35] O. Almora, I. Zarazua, E. Mas-Marza, I. Mora-Sero, J. Bisquert, G. Garcia-Belmonte, J. Phys. Chem. Lett. 2015, 6, 1645.

[36] Z. Xiao, Y. Yuan, Y. Shao, Q. Wang, Q. Dong, C. Bi, P. Sharma, A. Gruverman, J. Huang, Giant switchable photovoltaic effect in organometal trihalide perovskite devices. Nat. Mater. 2015, 14, 193.

[37] J. Yang, B. D. Siempelkamp, D. Liu, T. L. Kelly, ACS Nano 2015, 9, 1955.

[38] W. H. Nguyen, C. D. Bailie, E. L. Unger, M. D. McGehee, J. Am. Chem. Soc. 2014, $136,10996$.

[39] M. G. Walter, E. L. Warren, J. R. McKone, S. W. Boettcher, Q. Mi, E. A. Santori, N. S. Lewis, Chem. Rev. 2010, 110, 6446.

[40] R. Hezel, R. Meyer, A. Metz, Sol. Energy Mater. Sol. Cells 2001, 65, 311.

[41] A. Bera, K. Wu, A.Sheikh, E. Alarousu, O. F. Mohammed, and T. Wu, J. Phys. Chem. C 2014, 118, 28494. 


\section{WILEY-VCH}
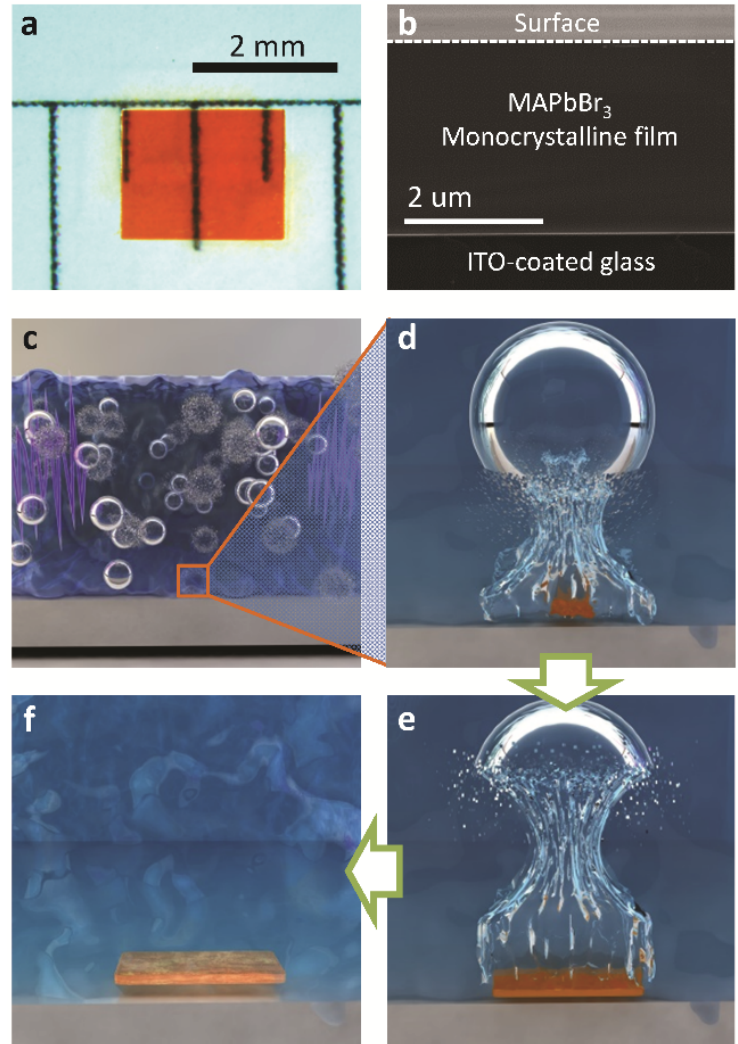

Figure 1. a) Optical image of the monocrystalline film. b) Cross-section SEM image of a freshly-cut monocrystalline film. c)-f) Illustrations of the CTAC mechanism on a microscopic scale. c) An ultrasonic pulse induces cavitation in the perovskite solution. d)-f) Collapse of the cavitation bubble at the neighborhood of a substrate is asymmetric and results in a highspeed jet towards the substrate, which is the origin of preferential lateral crystallization at the initial stage. 
WILEY-VCH
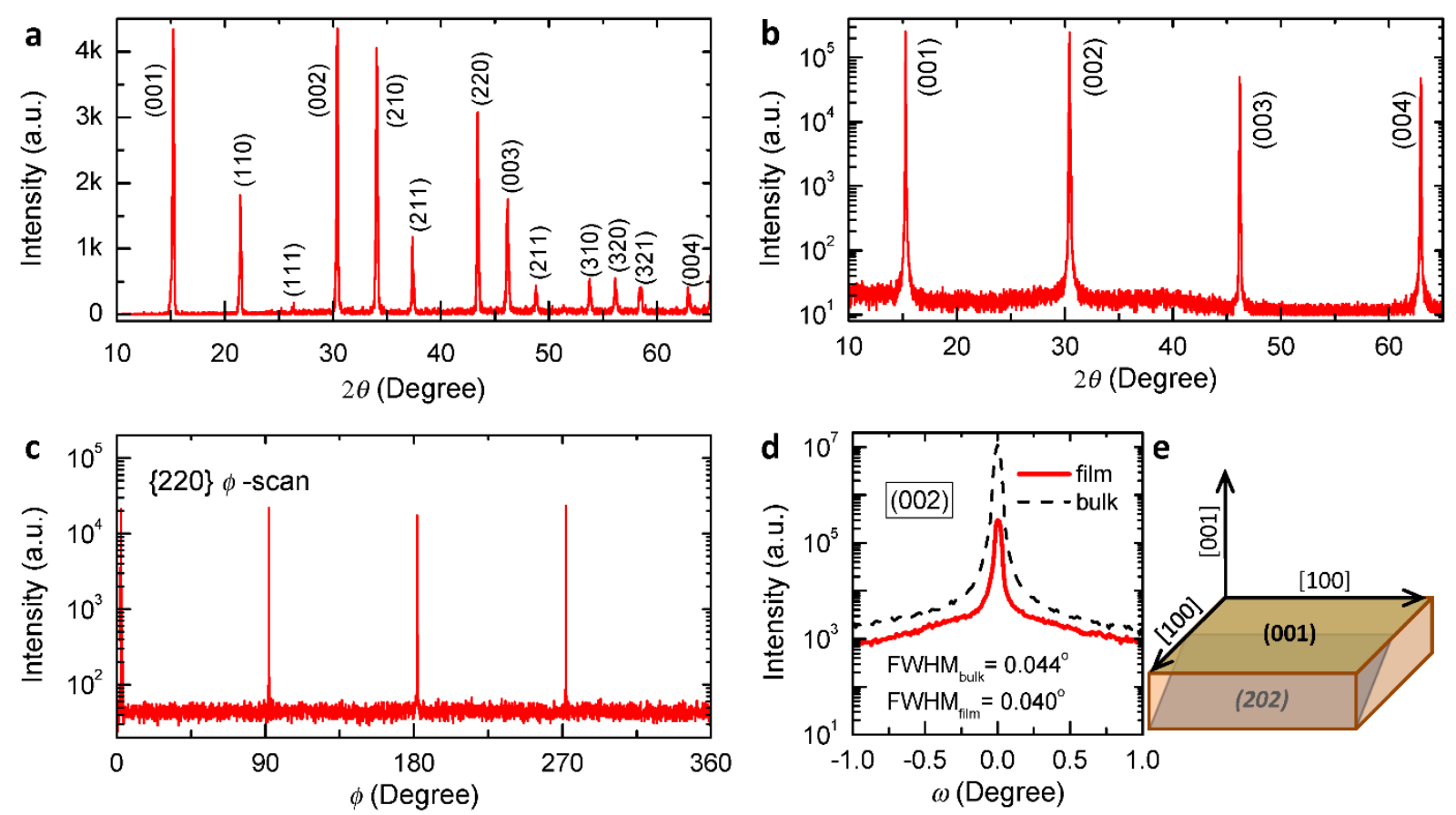

Figure 2. a) XRD pattern of grounded $\mathrm{MAPbBr}_{3}$ perovskite crystal powder. b) $\omega-2 \theta$ scan of the monocrystalline film reveals its (001) orientation. c) $\varphi$ scan of (110) diffraction shows a 4fold symmetry. d) Rocking curve of the (002) diffraction, which gives a FWHM of $0.040^{\circ}$. The rocking curve of a $\mathrm{MAPbBr}_{3}$ bulk crystal is also shown for comparison. e) A scheme of the (101) and (001) diffraction planes. 


\section{WILEY-VCH}

a
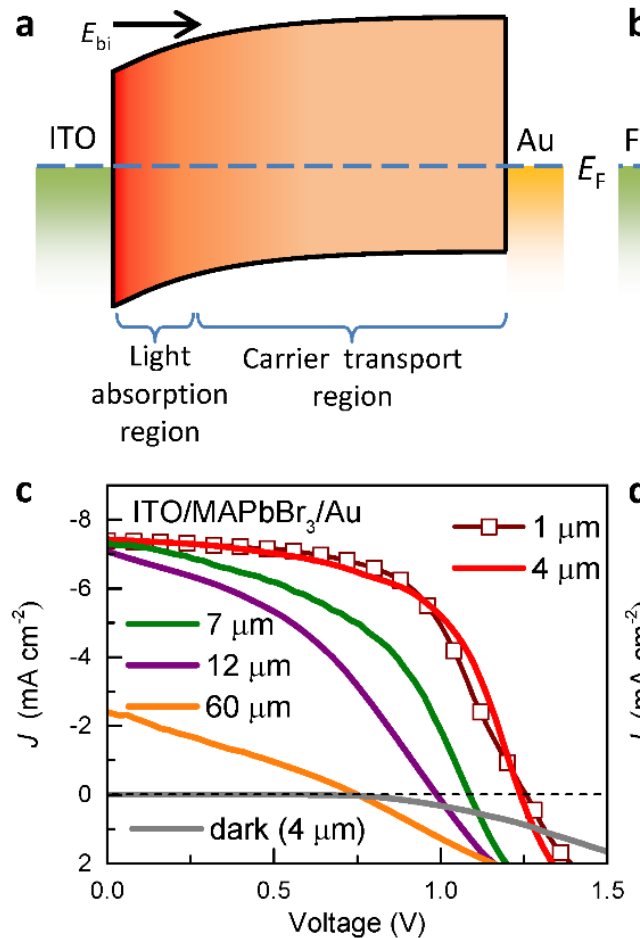
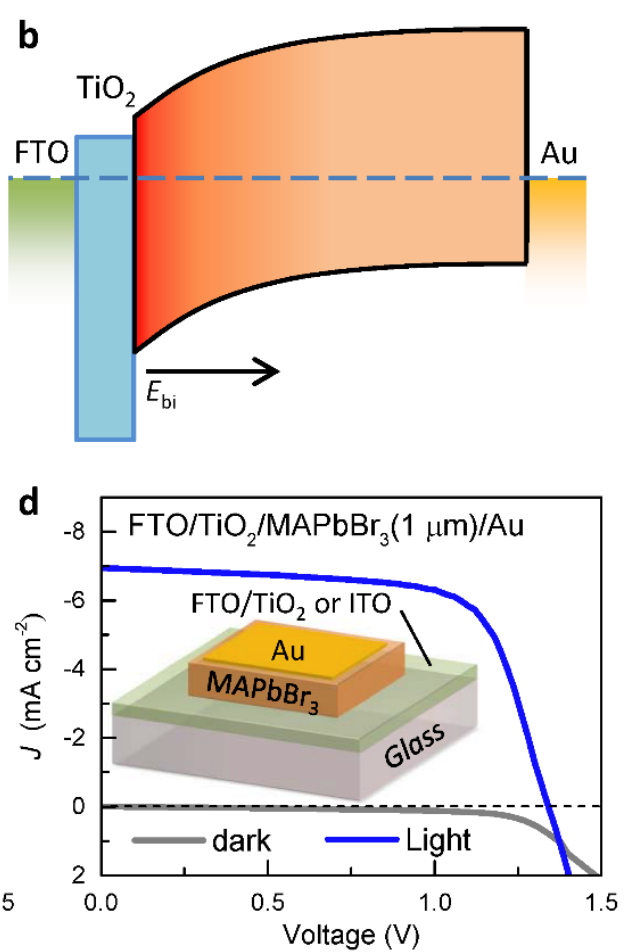

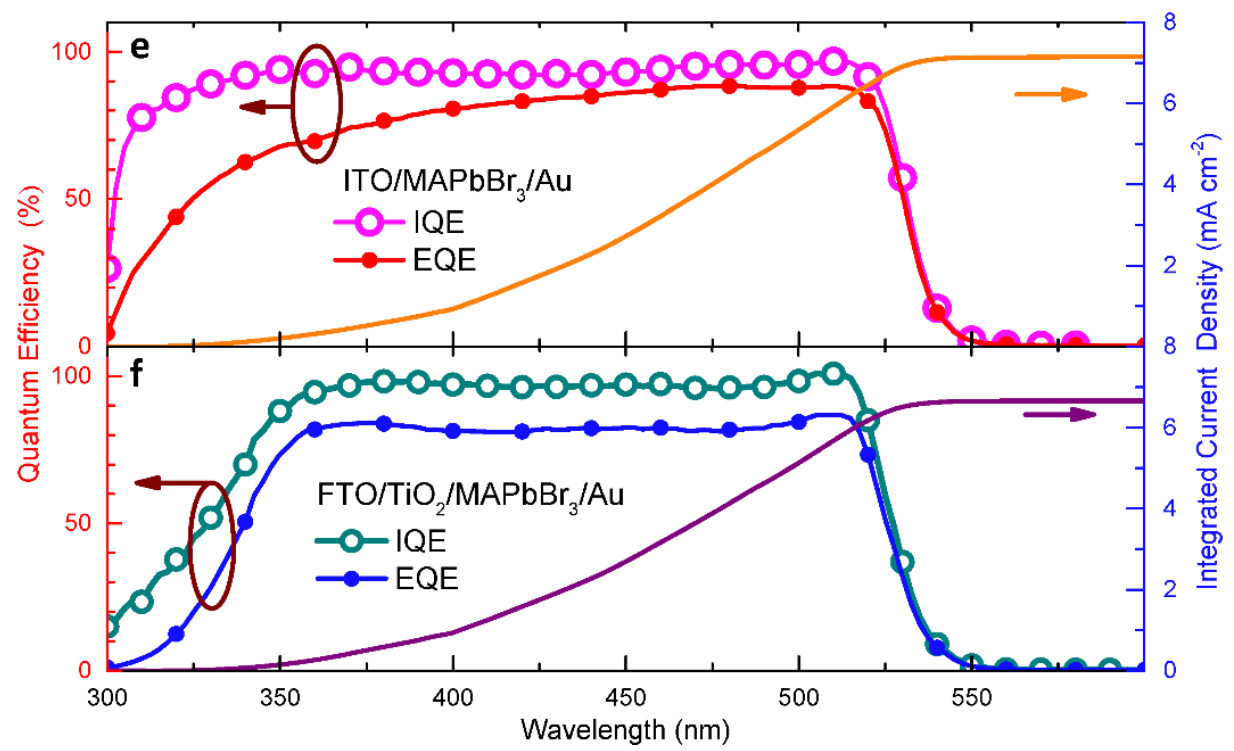

Figure 3. Band alignment of a) ITO/MAPbBr $3 / \mathrm{Au}$ and b) $\mathrm{FTO} / \mathrm{TiO}_{2} / \mathrm{MAPbBr}_{3} / \mathrm{Au}$. The dark orange color in the $\mathrm{MAPbBr}_{3}$ band diagram indicates the photocarriers are mostly generated near the interface of ITO/MAPbBr $3\left(\mathrm{TiO}_{2} / \mathrm{MAPbBr}_{3}\right)$ due to the large absorption coefficient. The direction of built-in field $E_{\mathrm{bi}}$ is also marked by the solid arrow. Dark and illuminated $J-V$ curves, wavelength-dependent EQE and IQE curves of c), e) ITO-based and d), f) $\mathrm{FTO} / \mathrm{TiO}_{2}-$ based monocrystalline solar cells, respectively. The quantum efficiency curves in e) and f) are measured from the device of $4 \mu \mathrm{m}$-thick crystal in c) and the device of $1 \mu \mathrm{m}$-thick crystal in d), respectively. d) The inset depicts an illustration of the device architecture. The integrated current densities calculated from EQE curves are also shown in e) and f). 

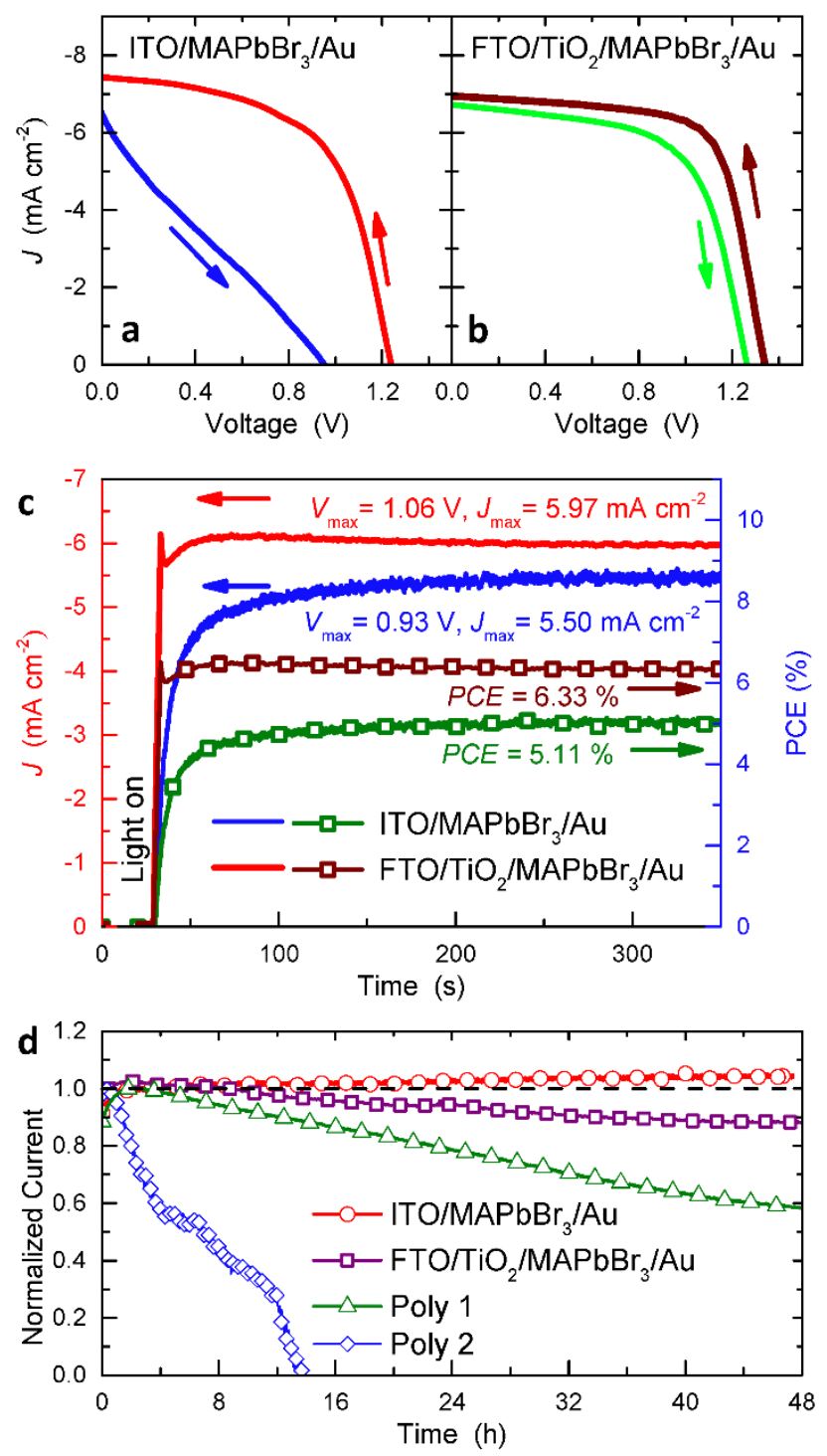

Figure 4. Reverse and forward (direction indicated by the solid arrow) scanned $J-V$ curves of a) ITO/MAPbBr $3 / \mathrm{Au}$ and b) $\mathrm{FTO} / \mathrm{TiO}_{2} / \mathrm{MAPbBr}_{3} / \mathrm{Au}$ under illumination. c) Steady-state photocurrents and PCEs of two monocrystalline devices at their corresponding $V_{\max }$. d) Stability test for two monocrystalline solar cells and two polycrystalline solar cells by monitoring the photocurrents at their corresponding $V_{\max }$ under continuous illumination of 1 sun for over 48 hours. 


\section{WILEY-VCH}

Table 1. Photovoltaic parameters extracted from the illuminated $J-V$ curves (reverse scanned) of monocrystalline solar cells with various $\mathrm{MAPbBr}_{3}$ film thicknesses.

\begin{tabular}{|c|c|c|c|c|c|}
\hline Device Structure & $\begin{array}{c}\text { Film thickness } \\
{[\mu \mathrm{m}]}\end{array}$ & $\begin{array}{l}V_{\mathrm{OC}} \\
{[\mathrm{V}]}\end{array}$ & $\begin{array}{c}J_{\mathrm{SC}} \\
{\left[\mathrm{mA} \mathbf{~ c m}^{-2}\right]}\end{array}$ & FF & $\begin{array}{l}\text { PCE } \\
{[\%]}\end{array}$ \\
\hline \multirow[t]{5}{*}{ ITO/perovskite/Au } & 1 & 1.25 & 7.39 & 0.59 & 5.49 \\
\hline & 4 & 1.24 & 7.42 & 0.58 & 5.37 \\
\hline & 7 & 1.11 & 7.19 & 0.46 & 3.70 \\
\hline & 12 & 1.03 & 7.09 & 0.39 & 2.82 \\
\hline & 60 & 0.94 & 2.27 & 0.31 & 0.65 \\
\hline FTO/TiO $/$ $_{2} /$ perovskite/Au & 1 & 1.36 & 6.96 & 0.69 & 6.53 \\
\hline
\end{tabular}




\section{WILEY-VCH}

The table of contents entry

High-quality, twin-free perovskite monocrystalline films are successfully grown by cavitation triggered asymmetric crystallization method. These films enable a simple cell structure, ITO/ $\mathrm{CH}_{3} \mathrm{NH}_{3} \mathrm{PbBr}_{3} / \mathrm{Au}$, with near $100 \%$ IQE, promising power conversion efficiencies (PCEs) $>5 \%$, and superior stability for prototype cells. Furthermore, the monocrystalline devices based on a hole transporter-free device structure yield PCEs of $6.5 \%$, the highest among other similarly structured $\mathrm{CH}_{3} \mathrm{NH}_{3} \mathrm{PbBr}_{3}$ solar cells to date.

Keyword: hybrid perovskites, single crystals, hole-transporter free solar cells

Wei Peng, Lingfei Wang, Banavoth Murali, Kang-Ting Ho, Ashok Bera, Namchul Cho, Chen-Fang Kang, Victor M. Burlakov, Jun Pan, Lutfan Sinatra, Chun Ma, Wei Xu, Dong Shi, Erkki Alarousu, Alain Goriely, Jr -Hau He, Omar F. Mohammed, Tom Wu, and Osman M. Bakr*

Solution-Grown Monocrystalline Hybrid Perovskite Films for Hole-Transporter Free Solar Cells

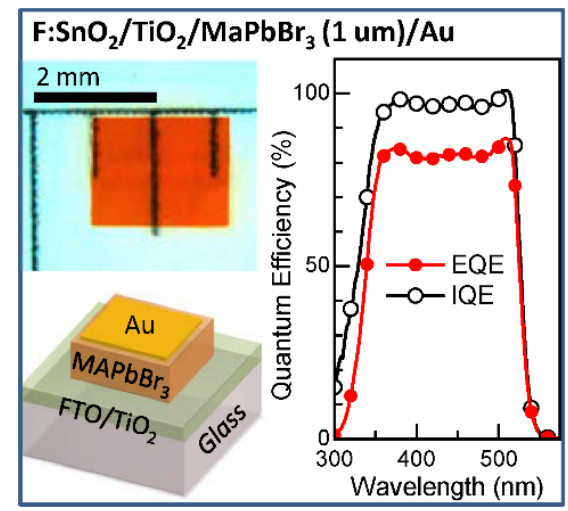




\section{WILEY-VCH}

Copyright WILEY-VCH Verlag GmbH \& Co. KGaA, 69469 Weinheim, Germany, 2013.

\section{Supporting Information}

Solution-Grown Monocrystalline Hybrid Perovskite Films for Hole-Transporter Free Solar Cells

Wei Peng, Lingfei Wang, Banavoth Murali, Kang-Ting Ho, Ashok Bera, Namchul Cho, ChenFang Kang, Victor M. Burlakov, Jun Pan, Lutfan Sinatra, Chun Ma, Wei Xu, Dong Shi, Erkki Alarousu, Alain Goriely, Jr-Hau He, Omar F. Mohammed, Tom Wu, and Osman M. Bakr* 


\section{WILEY-VCH}
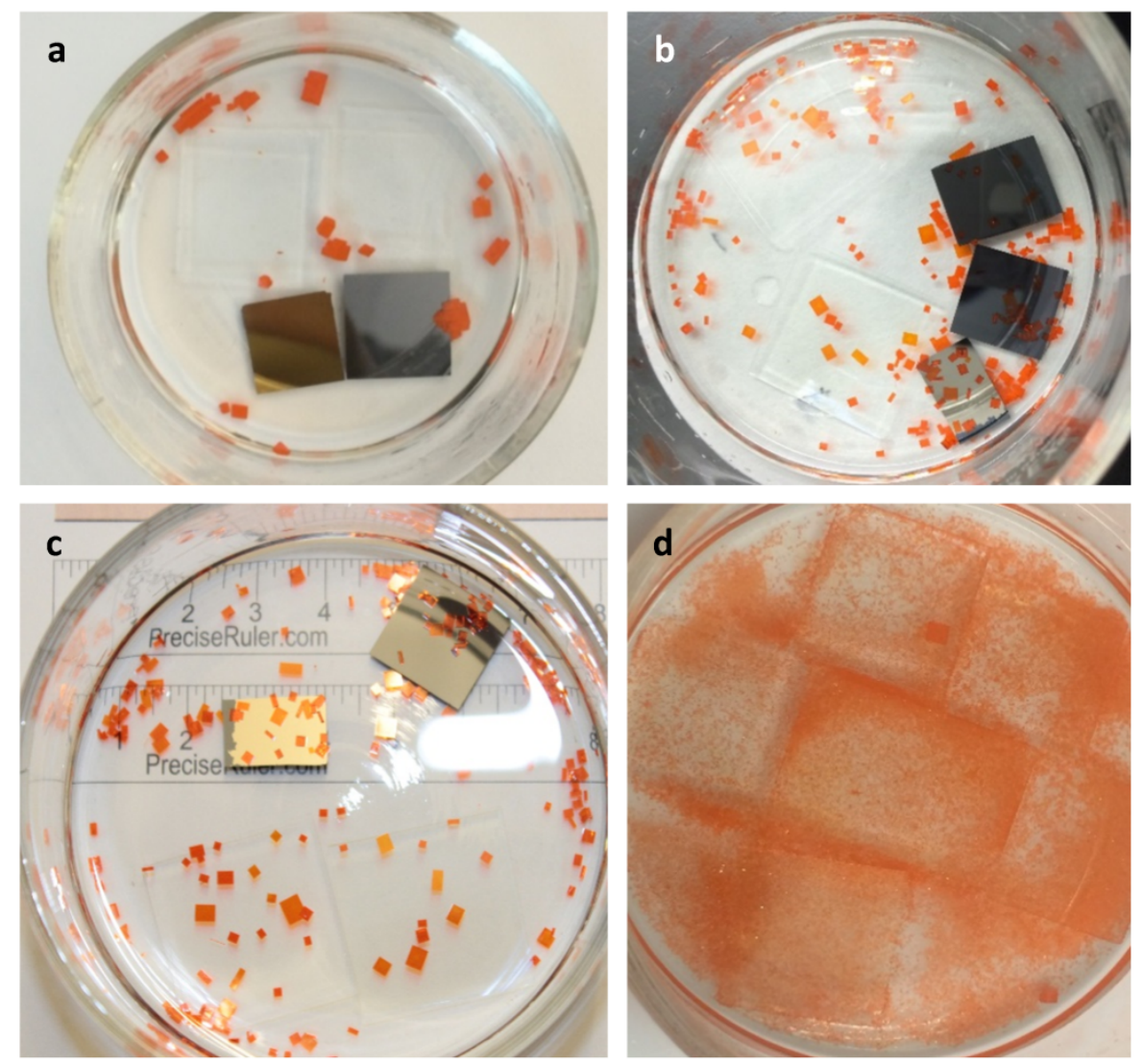

Figure S1. a) Optical photograph of $\mathrm{MAPbBr}_{3}$ single crystals grown by the antisolvent vapor diffusion crystallization method. The $\mathrm{MAPbBr}_{3}$ monocrystalline films grown on $b$ ) silicon wafer, ITO-coated glass and platinum-coated silicon wafer, and c) glass and gold-coated silicon wafer. d) Optical photograph of enormous tiny crystals grown by triggering the ultrasonic pulse at deep supersaturation stage. 


\section{WILEY-VCH}

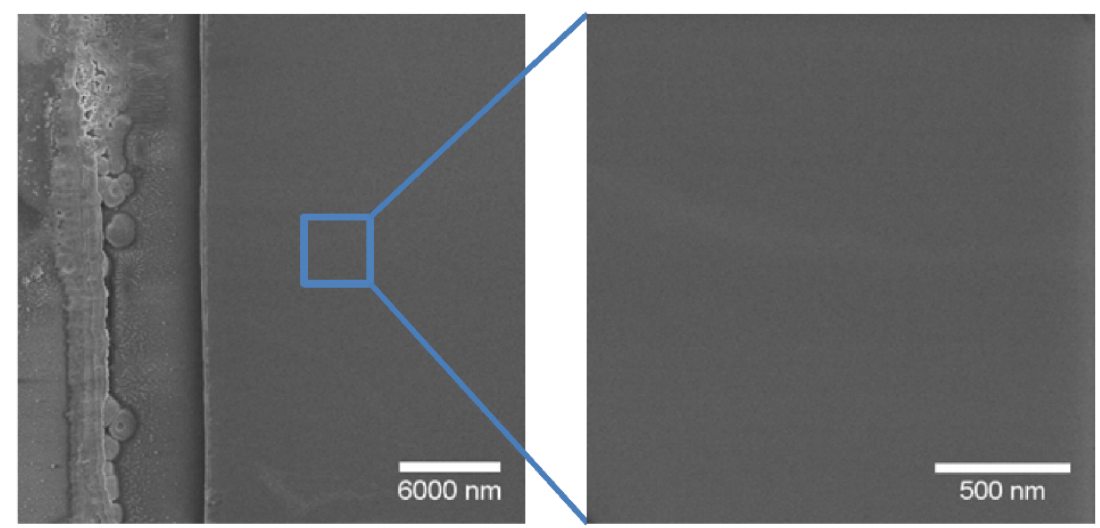

Figure S2. Top-view SEM images of $\mathrm{MAPbBr}_{3}$ monocrystalline films grown on substrates under different magnifications. The left image was taken from the edge of the monocrystalline film to increase contrast.

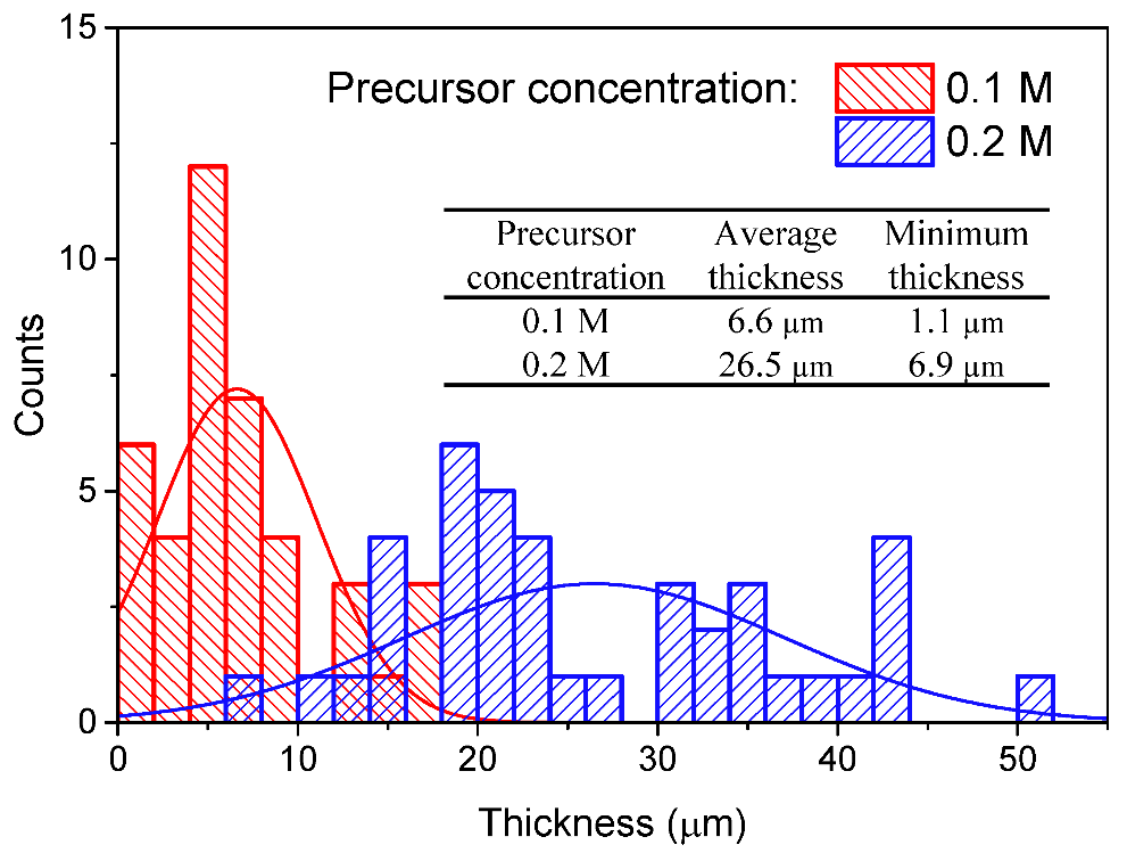

Figure S3. Statistic histograms of $\mathrm{MAPbBr}_{3}$ monocrystalline film thicknesses with different precursor concentrations. The average thickness and minimum thickness are also shown in the inset table.

We evaluated the dependence of film thickness on precursor concentrations by comparing approximately 50 discrete as-grown films from precursors of two concentrations, $0.1 \mathrm{M}$ and $0.2 \mathrm{M}$, after a fixed post-sonication growth time of $10 \mathrm{~h}$. As shown in Figure S3, the minimum (average) film thickness decreased from $6.1 \mu \mathrm{m}$ to $2.7 \mu \mathrm{m}(22.6 \mu \mathrm{m}$ to $8.9 \mu \mathrm{m})$ by reducing the concentration from $0.2 \mathrm{M}$ to $0.1 \mathrm{M}$. This dependence is related to a higher rate of crystal growth in a more concentrated solution. 


\section{WILEY-VCH}

\section{Space-charge-limited current analysis}

The current-voltage $(I-V)$ measurements were carried out on the $\mathrm{Au} / \mathrm{MAPbBr} / \mathrm{Au}$ monocrystalline sandwich structure. As shown in Figure S4, the logarithmic plot of $I-V$ curve clearly exhibit a trap-controlled space-charge-limited current (SCLC) behavior, including three distinct regions with different slopes. ${ }^{[1]}$ At small voltages, the device shows linear current response, i.e. Ohmic conduction. Further increasing the voltage, the current exhibits a rapid nonlinear rise (the onset voltage is defined as $\left.V_{\mathrm{TFL}}\right)$ with $I \propto V^{\mathrm{n}}(\mathrm{n}>3)$ at the trap-filled limit (TFL) regime. In the region of high voltages, a quadratic response, i.e. $I \propto V^{2}$, was observed, which follows the Child's law. The concentrations of in-gap deep trap states $\left(N_{\mathrm{dt}}\right)$ can be estimated by the equation $V_{\mathrm{TFL}}=e N_{\mathrm{dt}} d^{2} /\left(2 \varepsilon \varepsilon_{0}\right)$, where $d$ is the film thickness, $e$ and $\varepsilon_{0}$ represent the elementary charge and vacuum permittivity, respectively. The relative dielectric constant of $\mathrm{MAPbBr}_{3} \varepsilon$ is estimated as $25.5 .{ }^{[2]}$ As a result, the $N_{\mathrm{dt}}$ was calculated as $(1.39 \pm$ $0.21) \times 10^{11} \mathrm{~cm}^{-3}$.
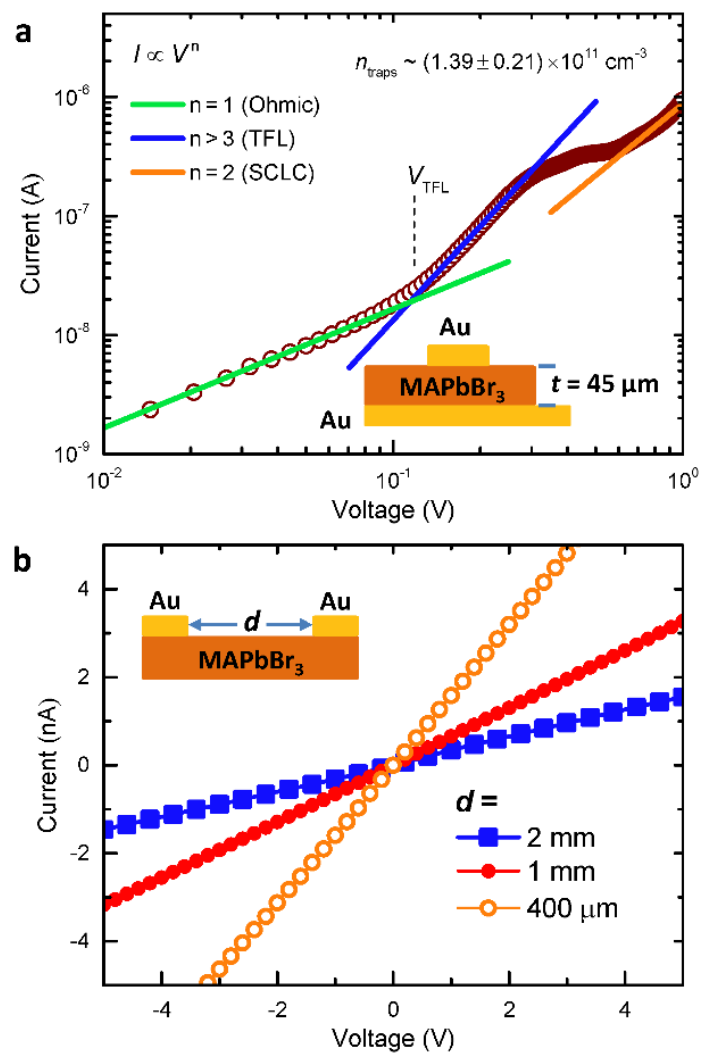

Figure S4. a) $I-V$ curve measured from the $\mathrm{Au} / \mathrm{MAPbBr} 3 / \mathrm{Au}$ monocrystalline sandwich structure (inset). Different regions of the logarithmic $I-V$ plot are linearly fitted and the $V_{\text {TFL }}$ are also marked in the plot. b) $I-V$ curves of a lateral $\mathrm{Au} / \mathrm{MAPbBr} 3 / \mathrm{Au}$ structure with varying distances between two Au electrodes, showing a linear characteristic. 


\section{WILEY-VCH}

\section{Photoluminescence and absorption measurements}

The existence of surface states in the monocrystalline film was corroborated by the absorption and photoluminescence (PL) spectra of the film. As shown in Figure S5a, the absorption peak at $529 \mathrm{~nm}$ and PL peak at $539 \mathrm{~nm}$ agree well with those captured from polycrystalline thin films (Figure S5b), while absorption edge at $553 \mathrm{~nm}$ and PL at $555 \mathrm{~nm}$ match those of bulk crystals. ${ }^{[2]}$ According to our previous experience, collecting the PL and absorption spectra of bulk single crystals while keeping them in the mother liquor could avoid the crystal surface being corroded and reconstructed. However, this set-up was difficult to achieve in the situation of characterizing monocrystalline films grown on substrates. Interestingly, a resonance feature is observable over the PL peak at $555 \mathrm{~nm}$, which should come from the Fabry-Pérot interference of the direct emission light of the bulk and the emission of light reflected by the substrate. ${ }^{[3]}$ This phenomenon further confirms the uniform nature of the monocrystalline films.

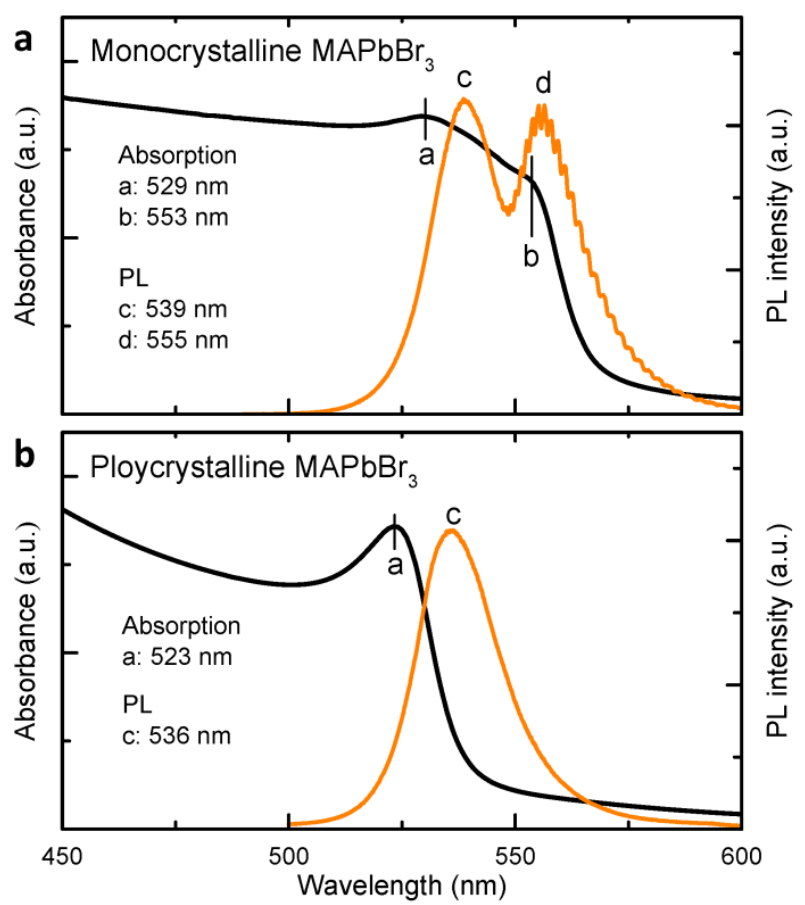

Figure S5. PL and optical absorption curves measured from $\mathrm{MAPbBr}_{3}$ a) monocrystalline film and b) polycrystalline film. 


\section{WILEY-VCH}

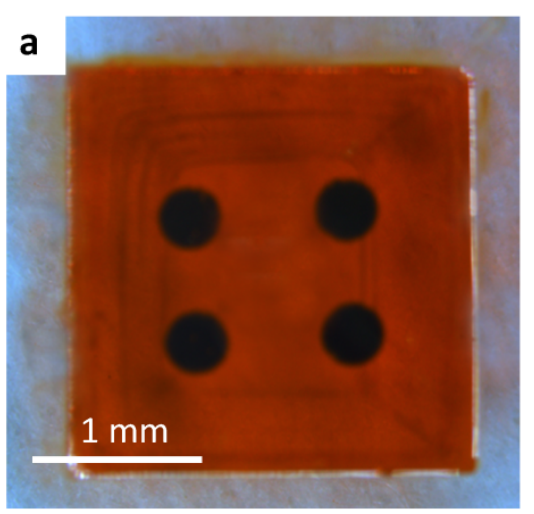

Without aperture mask

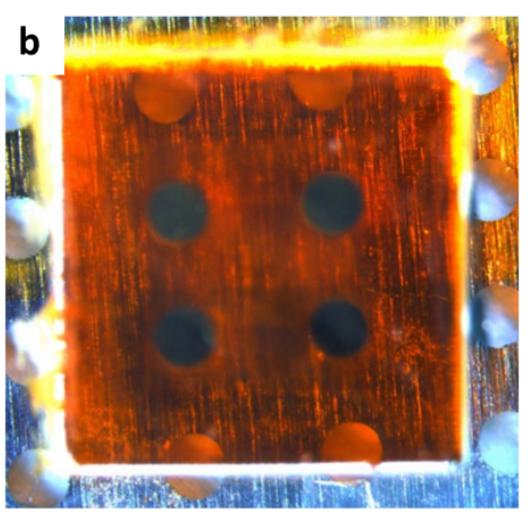

With aperture mask

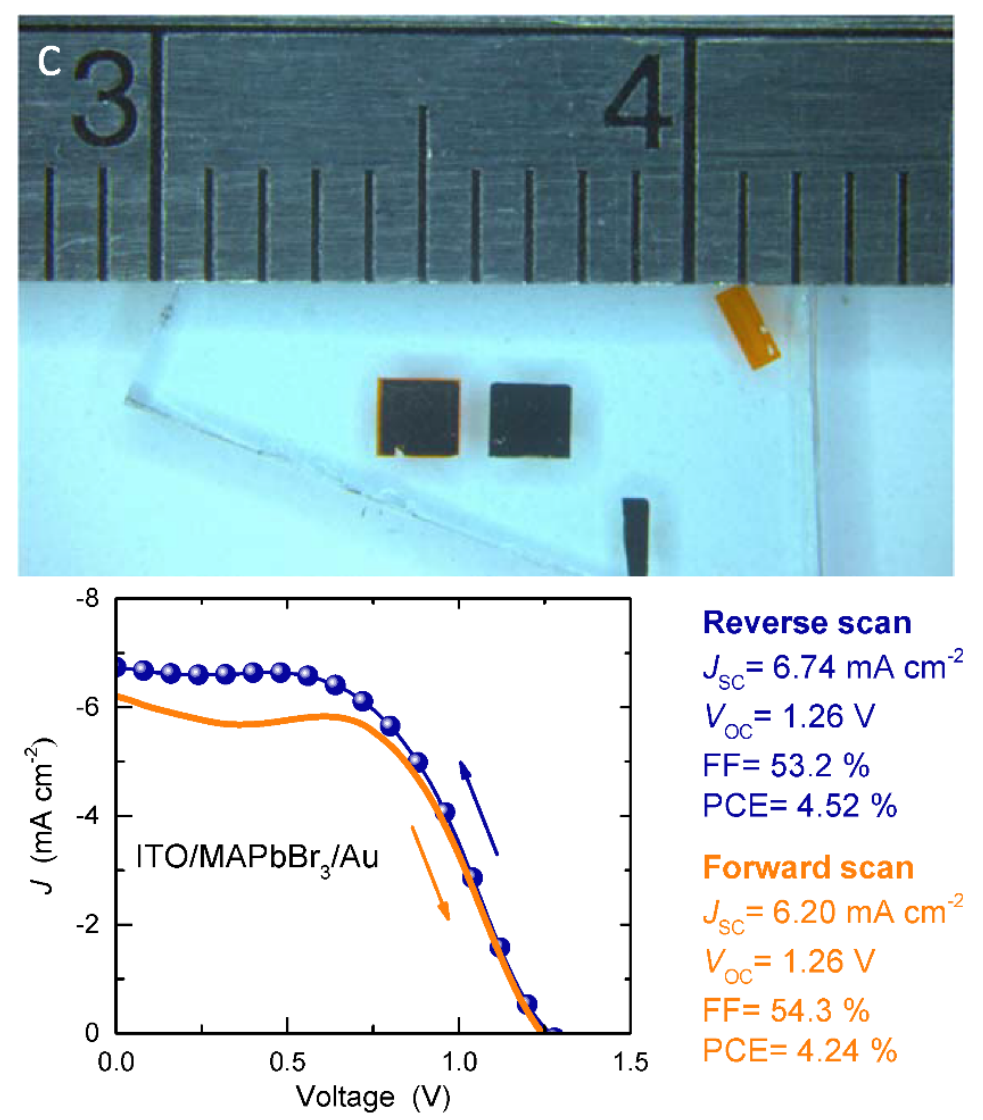

Figure S6. Photographs of one monocrystalline solar cell devices a) without and b) with the aperture mask; c) the photo of a $\sim 2 \times 2 \mathrm{~mm}^{2}$ device and its corresponding $J-V$ curve with the photovoltaic parameters also shown. The thickness of the monocrystalline film is $5 \mu \mathrm{m}$. 


\section{WILEY-VCH}

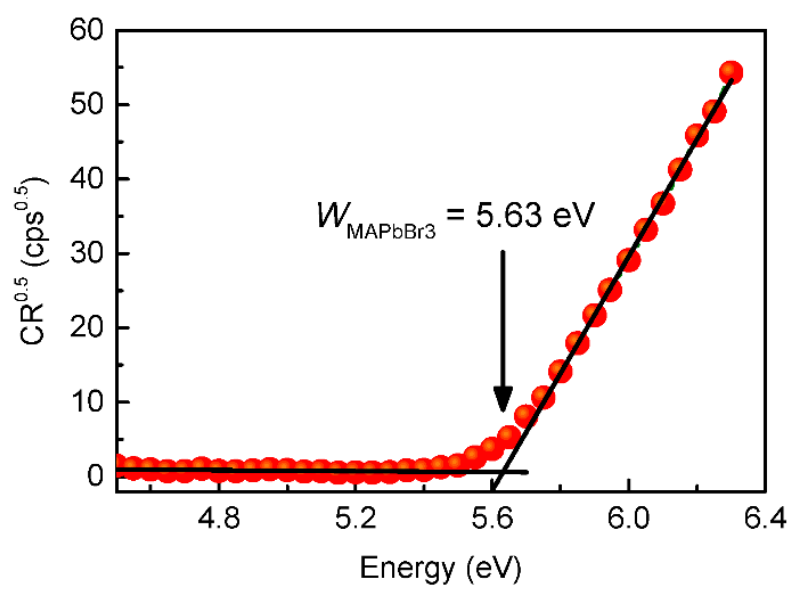

Figure S7. Photoelectron spectroscopy of $\mathrm{MAPbBr}_{3}$ monocrystalline films in air, showing a work function of $5.63 \mathrm{eV}$.
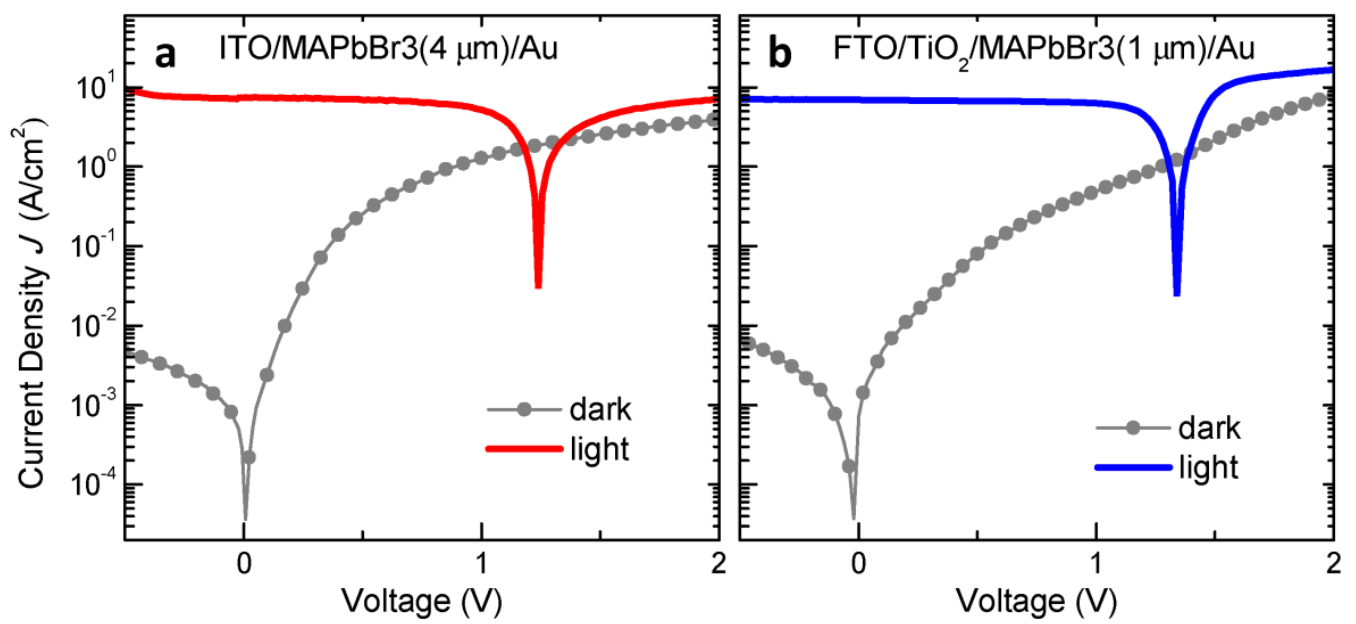

Figure S8. $J-V$ curves of a) ITO/monocrystalline $\mathrm{MAPbBr}_{3} / \mathrm{Au}$ and b) $\mathrm{FTO} / \mathrm{TiO}_{2} /$ monocrystalline $\mathrm{MAPbBr}_{3} / \mathrm{Au}$ in log scale under dark and illumination. 


\section{WILEY-VCH}
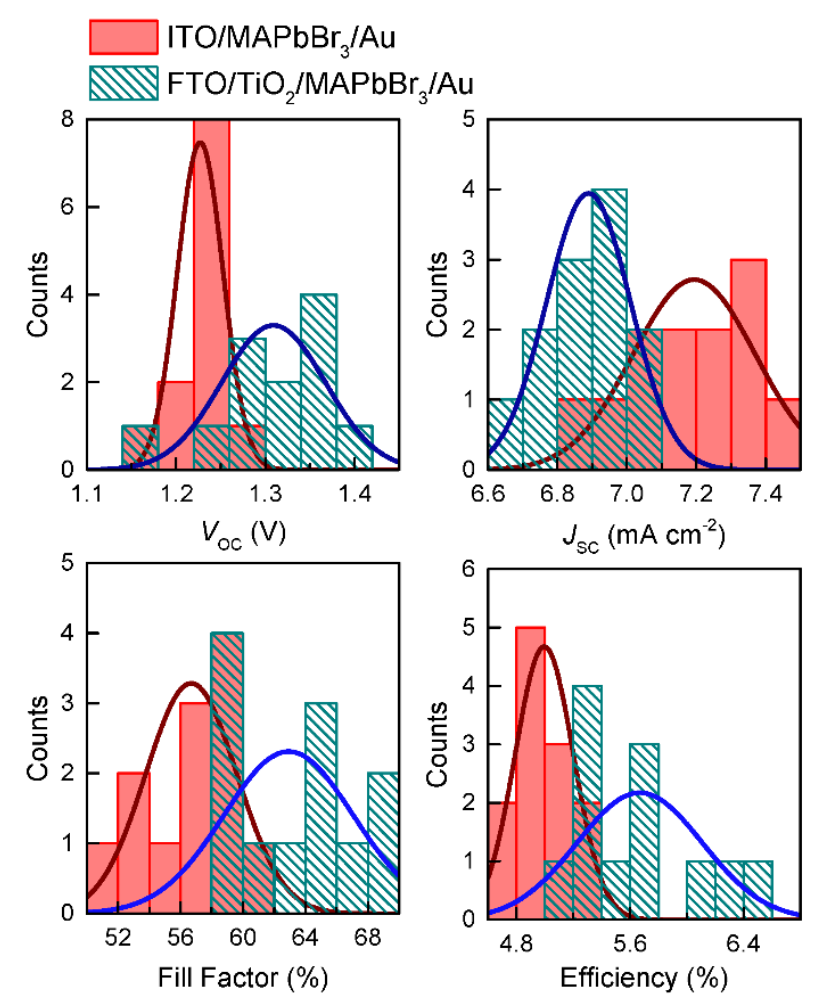

Figure S9. Statistical histograms of $\mathrm{MAPbBr}_{3}$ monocrystalline solar cell parameters extracted from 12 devices for each type. The thicknesses of monocrystalline films for ITO-based and $\mathrm{FTO} / \mathrm{TiO}_{2}$-based solar cells are $\sim 4 \mu \mathrm{m}$ and $<4 \mu \mathrm{m}$, respectively. 


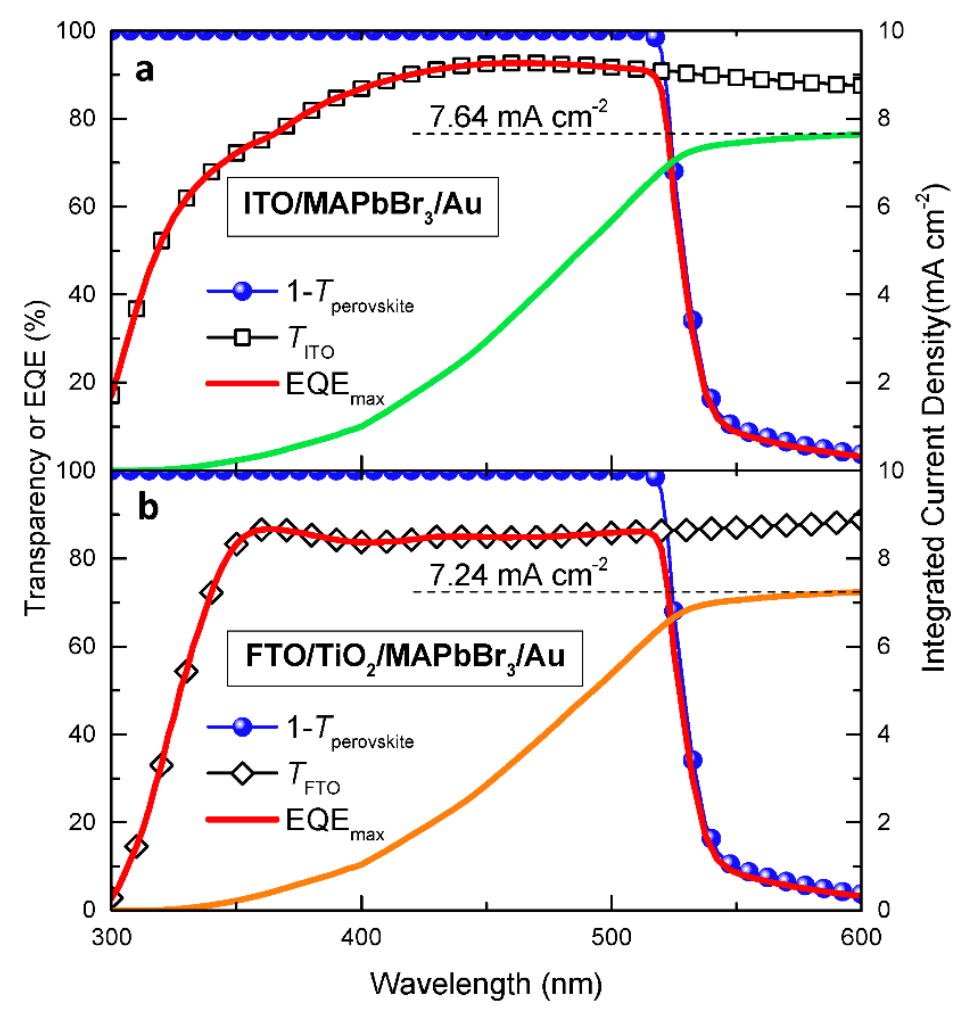

Figure S10. Wavelength-dependent transparency of a) bare ITO glass $\left(T_{\text {ITO }}\right)$ and b) FTO glass $\left(T_{\mathrm{FTO}}\right)$. The transmission of $\mathrm{MAPbBr}_{3}$ single crystal films $\left(T_{\text {perovskite }}\right)$ was also plotted in the form of $1-T_{\text {perovskite }}$ to define the onset of $\mathrm{EQE}_{\max }$ curve, i.e. theoretically upper limit of $\mathrm{EQE}$, for two different structured perovskite monocrystalline devices. $\mathrm{EQE}_{\max }$ can be achieved only when the device can absorb all the photons that transmit through ITO or FTO substrates, convert them into electrons and then collect completely (i.e. 100\% IQE). Therefore the $\mathrm{EQE}_{\max }$ curve will overlap with the transparency curve of corresponding substrates at wavelengths below onset absorption, as presented in both a) and b). Hence, the theoretical integrated current density is calculated for both device structures: $7.64 \mathrm{~mA} \mathrm{~cm}^{-2}$ for ITO/MAPbBr $3 / \mathrm{Au}$ and $7.24 \mathrm{~mA} \mathrm{~cm}{ }^{-2}$ for $\mathrm{FTO} / \mathrm{TiO}_{2} / \mathrm{MAPbBr}_{3} / \mathrm{Au}$. 


\section{WILEY-VCH}

\section{Mott-Schottky analysis of $\mathrm{MAPbBr}_{3} / \mathrm{Au}_{\text {Schottky diode }}$}

The capacitance-voltage ( $C-V)$ measurements can be used to determine the built-in potential, depletion width and carrier concentration. ${ }^{[4]}$ According to the depletion approximation, the junction capacitance is given by

$$
C=A \varepsilon \varepsilon_{0} / W
$$

$A$ is the device area, $\varepsilon$ and $\varepsilon_{0}$ refer to dielectric constant (25.5 for $\left.\mathrm{MAPbBr}_{3}\right)$ and the vacuum permittivity, respectively. The depletion width, $W$, of a Schottky junction is given by

$$
W=\sqrt{\frac{2 \varepsilon \varepsilon_{0}\left(V_{b i}-V\right)}{e N_{D}}}
$$

where $e$ is the elementary charge, $N_{\mathrm{D}}$ is the dopant density, $V$ is the applied bias and $V_{\mathrm{bi}}$ is the built-in potential. From Equation S1 and S2 we can get

$$
C^{-2}=\frac{2\left(V_{b i}-V\right)}{A^{2} e \varepsilon \varepsilon_{0} N_{D}}
$$

From which we express the dopant density as

$$
N_{D}=\frac{1}{A^{2}} \frac{2}{e \varepsilon \varepsilon_{0} \frac{d}{d V}\left(\frac{1}{c^{2}}\right)}
$$

The Mott-Schottky plot of $C^{-2}$ versus $V$ is shown in Figure S11. According to Equation S3, a $V_{\mathrm{bi}}$ of $\sim 1.2 \mathrm{eV}$ can be obtained from the linear extrapolation of $C^{-2}-V$ curve to zero. The selfdopant density of $7.15 \times 10^{13} \mathrm{~cm}^{-3}$ can be calculated by the linear fitting slope of $C^{-2}-V$ curve. From Equation $\mathrm{S} 2$ the depletion width $\mathrm{W}$ can be calculated as $6.88 \mu \mathrm{m}$.

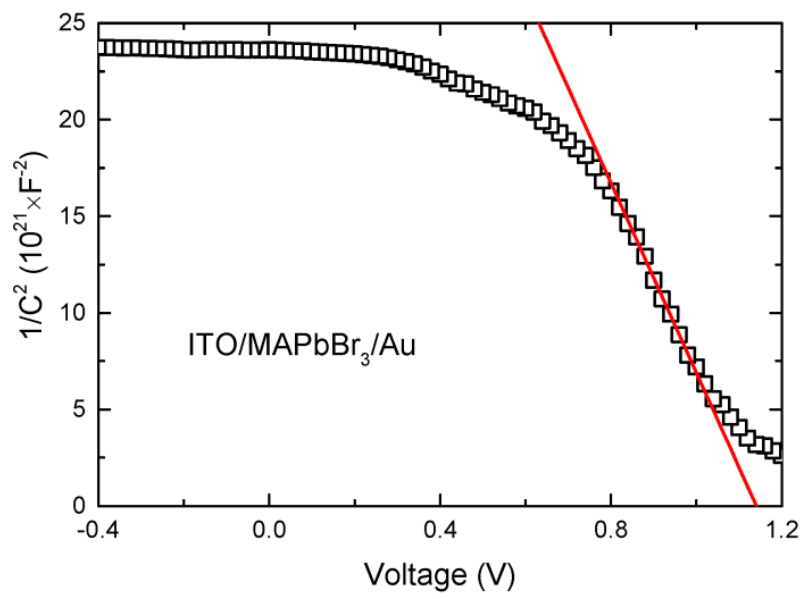

Figure S11. Mott-Schottky plot of the $C-V$ measurement from the ITO/monocrystalline $\mathrm{MAPbBr} / \mathrm{Au}$ solar cell and the fitting curve at the linear regime. 


\section{WILEY-VCH}
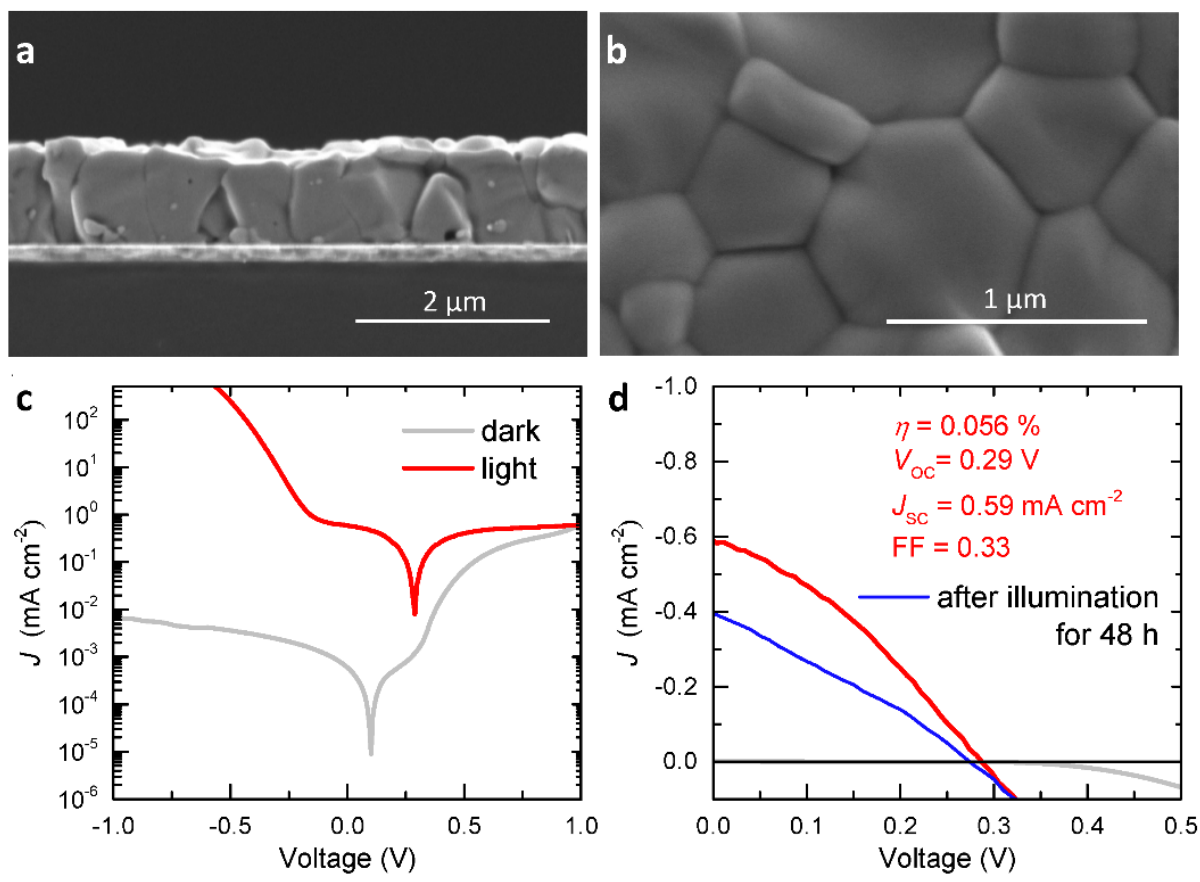

Figure S12. a) Cross-sectional and b) top-morphology SEM images measured from the polycrystalline $\mathrm{MAPbBr}_{3}$ film grown on ITO-coated glass. The film consists of an entire monolayer of grains with smooth surface morphology and full coverage. The dark and illuminated (AM1.5, $100 \mathrm{~mW} \mathrm{~cm}^{-2}$ ) $J-V$ curves of the ITO/polycrystalline $\mathrm{MAPbBr}_{3} / \mathrm{Au}$ device in c) log-scale and d) linear scale. The corresponding illuminated $J$ - $V$ curve after 48 hour continuous illumination is also shown in d). 

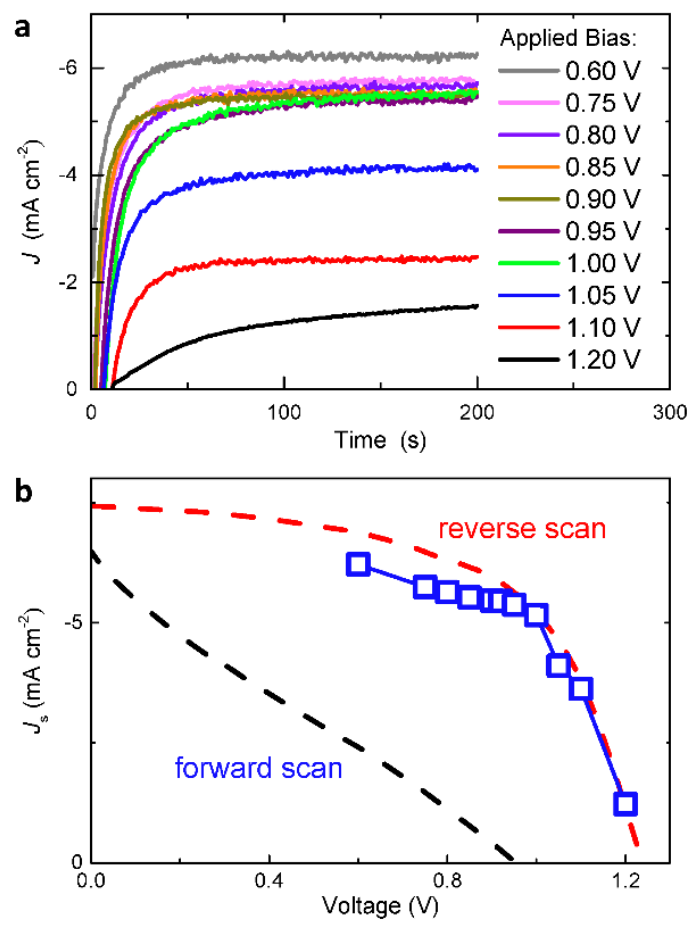

Figure S13. a) Transient current curves under various biases of the cell ITO/monocrystalline $\mathrm{MAPbBr} / \mathrm{Au}$ and $\mathrm{b}$ ) the corresponding stable photocurrent (blue open square) agrees well with the reverse scanned $J-V$ curve.

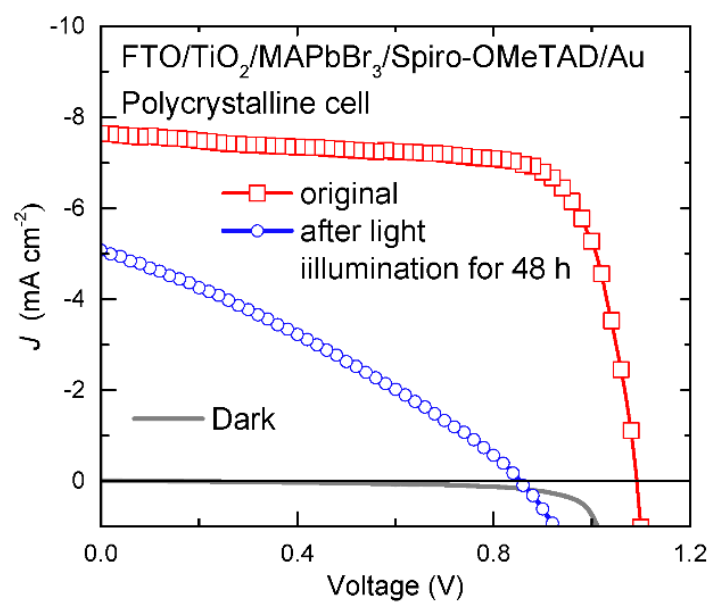

Figure S14. Dark and illuminated $J-V$ curves measured from the meso-structured solar cell. The illuminated $J-V$ curve after 48 -hour continuous illumination is also shown. 


\section{WILEY-VCH}
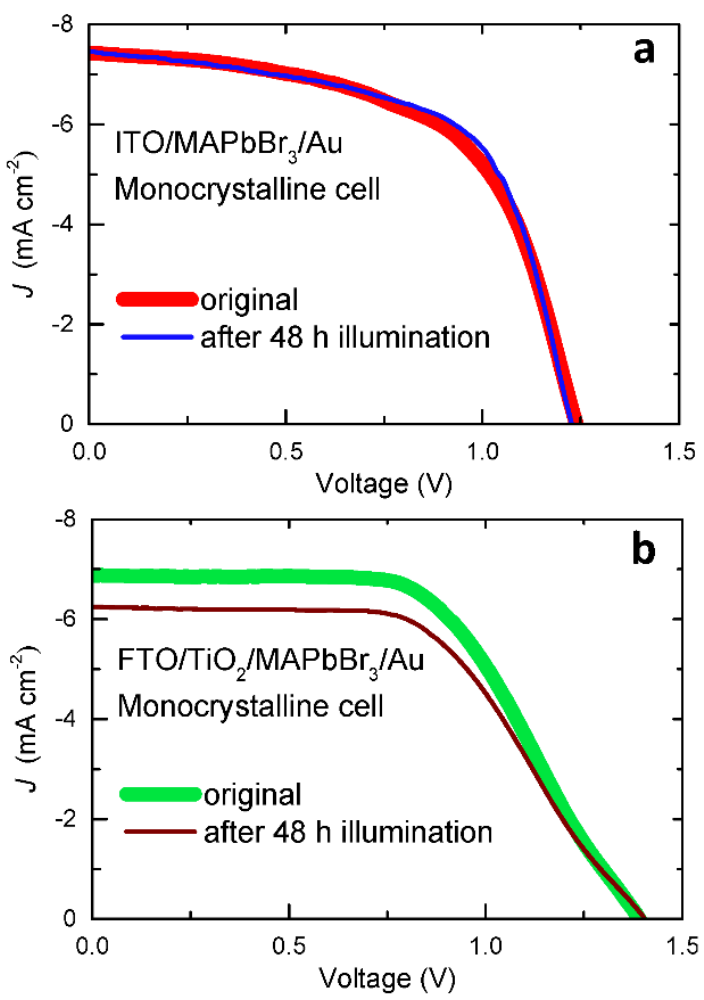

Figure S15. The illuminated $J-V$ curves of a) ITO/monocrystalline $\mathrm{MAPbBr}_{3} / \mathrm{Au}$ and b) $\mathrm{FTO} / \mathrm{TiO}_{2} /$ monocrystalline $\mathrm{MAPbBr}_{3} / \mathrm{Au}$ solar cells measured before and after 48 -hour continuous illumination. 


\section{WILEY-VCH}

\section{Reference}

1. M. A. Lampert, Phys. Rev. 1956, 103, 1648.

2. D. Shi, V. Adinolfi, R. Comin, M. Yuan, E. Alarousu, A. Buin, Y. Chen, S. Hoogland, A. Rothenberger, K. Katsiev, Y. Losovyj, X. Zhang, P. A. Dowben, O. F. Mohammed, E. H. Sargent, O. M. Bakr, Science 2015, 347, 519.

3. C. Hums, T. Finger, T. Hempel, J. Christen, A. Dadgar, A. Hoffmann, A. Krost, J.Appl. Phys. 2007, 101, 033113.

4. J. M. Luther, M. Law, M. C. Beard, Q. Song, M. O. Reese, R. J. Ellingson, and A. J. Nozik, Nano Lett. 2008, 8, 3488. 